\title{
Analysis of the influence of the plasma thermodynamic regime in the spectrally resolved and mean radiative opacity calculations of carbon plasmas in a wide range of density and temperature
}

\author{
J.M. Gil ，R. Rodriguez ，P. Martel ，R. Florido ，J.G. Rubiano ，M.A. Mendoza ， \\ E. Minguez
}

\begin{abstract}
A B S T R A C T
In this work the spectrally resolved, multigroup and mean radiative opacities of carbon plasmas are calculated for a wide range of plasma conditions which cover situations where corona, local thermodynamic and non-local thermodynamic equilibrium regimes are found. An analysis of the influence of the thermodynamic regime on these magnitudes is also carried out by means of comparisons of the results obtained from collisional-radiative, corona or Saha-Boltzmann equations. All the calculations presented in this work were performed using ABAKO/RAPCAL code.
\end{abstract}

\section{Introduction}

The role of the radiative properties is known to be of decisive importance to many research fields of plasma physics, such as astrophysics or nuclear fusion both inertial and magnetic confinement, since they are essential to explain and analyze the experiments or observations and a key radiative property is the opacity, which quantifies how transparent or opaque the plasma is to the radiation. Thus, in astrophysics, the opacities of stellar mixtures control the energy transfer in stars, which affects the stellar structure and evolution [1], and rule the levitation of metals in stellar interiors [2]. In the research of inertial confinement fusion the opacities are essential in the design of hohlraum walls. Furthermore, they are involved in radiative-hydrodynamic simulations where the energy of the matter and the radiation are of the same order, and, also, the theoretical calculations of opacity also provide a method to the temperature and density diagnostics of hot plasmas.

Due to its relevance, research on radiative opacities has greatly developed during the past several decades, though most of them were focused on Local Thermodynamic Equilibrium (LTE) regime [3-8]. However, NonLocal Thermodynamic Equilibrium (NLTE) plasmas are found in a wide range of density and temperature conditions as for example in inertial confinement fusion (both direct and indirect-drive fusion schemes), in magnetic confinement fusion (both in tokamaks and stellarator devices), $z$-pinch plasmas, coherent X-ray sources, ultrashort pulse laser-produced plasmas or stellar atmospheres. On the hydrodynamic time scales, NLTE thermodynamic regime is favored when the plasma presents strong time and space gradients and hence there is not enough time for equilibrium to be achieved, or even in the 
case of homogeneous and time independent plasma, NLTE conditions exist if plasma is of finite size and the photon can escape from it.

In principle, the calculation of opacity for LTE plasmas is as similar as that for NLTE plasmas except that the calculation of the atomic level populations is different. In the first case, the level populations are obtained by solving the Saha-Boltzmann (SB) equations, and in the second one, the populations are obtained by solving the rate equations, being the so-called collisional-radiative model (CRM) an essential tool for these calculations. However, the complexity of the rate equations, which requires a huge amount of atomic data and collisional and radiative atomic processes, makes much more difficult to give accurate level populations and radiative properties for NLTE plasmas than for LTE ones. For this reason, during the recent years many groups have been developing NLTE radiative properties calculations and codes [7-26], and new models and results are always welcomed.

Carbon is one of the most important elements under investigation in several research areas: in astrophysics, due to its abundance in the stars; in magnetic nuclear fusion confinement, because it is likely to be a major plasma-facing wall component in the international experimental reactor (ITER) and it is present as impurity in a lot of devices; and it plays a major role in inertial fusion scenarios, for example, in the direct-drive implosion cores where the deuterium target has a thickness plastic shells. In a previous work [27], we presented a systematic calculation of average ionization, ionic and level populations of the optically thin carbon plasma in a wide range of plasma conditions. We assumed that the comparison between the average ionization and ion and level populations calculated from collisional-radiative steady state (CRSS), Corona (C) and SB equations can provide information about the thermodynamic regime of the plasma, determining the plasma conditions where Corona equilibrium (CE), LTE or NLTE could be assumed. This work is a continuation of the previous one, since it is performed a study of spectrally resolved, multigroup and mean opacities of carbon plasmas. The interest in multigroup and mean opacities is related to the resolution of the radiative transport equation. It is well known that this equation is very complicated and, in general, one must approximate the radiative equation in order to obtain an analytical solution or to reduce the cost of the numerical solution. The aim of a large number of applications is to obtain numerical solution of the specific intensity in the space and time, and approximations on the angle and frequency dependence are commonly done. Usually, the angular dependence is represented by a spherical harmonic expansion in the low order angular or diffusion approach which is obtained when only the first two terms in the expansion are considered. There exist many methods to approximate the frequency dependence, and we can divide these into two families. The first one, the multigroup methods in which the frequency spectrum is discretized into $n$ frequency contiguous groups, assigning a frequency group to a given photon and a mean opacity for each frequency group from the average of the spectrally resolved opacity. Finally, the radiative transport equation is solve for each group. The second method is based on the key recognition that transport of photons depends on the value of the opacity not the value of the frequency. In this case one can discretize spectrally resolved opacity into $n_{b}$ opacity contiguous groups and reorganize the frequency spectra according to the value of the opacity. This creates disjoint sets of frequencies, the bins or pickets, with similar spectrally resolved opacity. Now we assign a mean opacity for each bin and solve the transport equation for each one. Both methods require a procedure or definition to obtain the mean opacity, which consist in the selection of the photon distribution function or weighting function, and this can of course be made by different ways. Special cases commonly used are the Planck mean opacity, which is appropriate in the time independent radiative transport in a LTE optically thin plasmas, or if the equilibrium diffusion approximation is considered, the Rosseland mean opacity.

The temperature and density conditions analyzed in this work were $10^{-12}-10^{-1} \mathrm{~g} \mathrm{~cm}^{-3}$ and $1-10^{3} \mathrm{eV}$, respectively. These ranges of plasma conditions cover LTE and NLTE thermodynamic regimes. This fact will allow us to provide the opacity data of carbon plasma in a wide range of plasma conditions and to study the influence of the thermodynamic regime into opacities by means of the comparisons between opacities calculated by using level populations obtained from CRSS and SB equations. The study will be carried out analysing spectrally resolved, multigroup and mean opacity magnitudes, we have assumed Planck and Rosseland multigroup and mean opacities, and all the calculations presented in this work were performed using the ABAKO/RAPCAL computational package $[22,26,28]$. The atomic data have been obtained under the detailed-level-accounting (DLA) approach including configuration mixing using FAC code [29]. In the next section, the theoretical model is briefly described. In Sections 3 and 4 results and conclusions are presented, respectively.

\section{Theoretical model}

The calculations in this work were performed using the computational package ABAKO/RAPCAL [28] which consists in two codes, ABAKO [26] and RAPCAL [21,22] devoted to the calculation of the plasma level populations and radiative properties, respectively. A brief description of both codes is given in the following.

\subsection{Atomic data and level populations}

In order to determine the plasma level populations, in ABAKO is implemented a collisional-radiative steady state (CRSS) model. Following the standard NLTE modeling approach, where an account of the existing atomic states is made and the microscopic (radiative and collisional) processes connecting these states are identified, a rate equation system describing the population density of the atomic states is built and solved, giving the population distribution. Therefore, to find the level population 
distribution the following system of rate equations is solved:

$\sum_{\zeta^{\prime} j} N_{\zeta^{\prime} j} \mathbb{R}_{\zeta^{\prime} j \rightarrow \zeta i}^{+}-\sum_{\zeta^{\prime} j} N_{\zeta_{i} i} \mathbb{R}_{\zeta_{i} \rightarrow \zeta^{\prime} j}^{-}=0$,

where $N_{\zeta i}$ is the population density of the atomic level $i$ of the ion with charge state $\zeta$. The terms $\mathbb{R}_{\zeta^{\prime} j \rightarrow \zeta i}^{+}$and $\mathbb{R}_{\zeta^{\prime} j \rightarrow \zeta i}^{-}$ take into account all the atomic processes which contribute to populate and depopulate the state $\zeta i$, respectively. In this paper no radiation-driven processes are explicitly considered. In ABAKO is assumed that the system has had enough time to thermalize and, therefore, both the electrons and ions have a Maxwell-Boltzmann type energy distribution. Furthermore, in ABAKO is also assumed that electron and ion temperatures are equal. Therefore, in the following, it will denote the plasma temperature by the electron temperature $T_{e}$.

The CRSS implemented in ABAKO is applied to low-tohigh $Z$ ions under a wide range of laboratory or astrophysical plasma conditions: CE, NLTE or LTE, optically thin and thick plasmas, homogeneous and non-homogeneous plasmas. A special care was taken during the development of this CRSS model to achieve an optimal equilibrium between accuracy and computational cost. Hence, it has been employed analytical expressions for the rate coefficients of the atomic processes included in the CRSS model, which yield a substantial saving of computational requirements, but providing satisfactory results in relation to those obtained from more sophisticated codes and experimental data as it has been proved in the last NLTE code comparison workshops [30-32]. The processes included in this CRSS model are the following: collisional ionization [33] and three-body recombination, spontaneous decay, collisional excitation [34] and deexcitation, radiative recombination [35], electron capture and autoionization [36]. Between brackets it has been added the references where from their approximated analytical rates coefficients have been acquired. The rates of the inverse processes are obtained through the detailed balance principle. It is worth pointing out that the autoionizing states are included explicitly. It has been shown that their contribution can be critical in the determination of the ionization balance, overall at low electron densities and high temperatures where $C E$ is achieved [37,38]. The cross section of the autoionization is evaluated using detailed balance principle from the electron capture cross section. This one is obtained from the collisional excitation cross section using a known approximation [36]. Finally, when LTE conditions are attained, the level populations in ABAKO can be also obtained by means of the SB equations.

Since the number of rate equations is huge due to the number of atomic levels involved, ABAKO makes use of the technique of sparse matrices to storage the non-zero elements of the coefficient matrix of the system, which implies substantial savings in memory requirement. For the matrix inversion iterative procedures have been used [28] because they entail much less memory than direct methods and they are also faster.

The atomic data employed in this work were obtained form FAC code [29]. The calculations were carried out in the DLA approach. The radiative transitions rates in FAC are calculated in the single multipole approximation, and in this work they were obtained in the electric dipole approach. Furthermore, configuration interactions within the levels belonging to the same non-relativistic configuration have been included since it has been shown that for carbon plasmas, when the less ionized ions are present, the effect of configuration interaction is noticeable [39]. In Table 1 it is shown the set of relativistic configurations and the numbers of resulting levels and line transitions included for each carbon ion in the calculation of the level populations and opacities. In the table $(n)^{w}$ denotes all the possible relativistic configurations that arise from the shell $n$ with $w$ bound electrons. Finally, the continuum lowering due to the influence of the plasma surrounding is also considered. In this work, this one is calculated by means of the expression due to Stewart and Pyatt [40]. Because of the inclusion of the continuum lowering, the kinetics equations must be solved iteratively, since the atomic data depend on the ionization balance.

\subsection{Spectrally resolved and mean radiative opacities}

RAPCAL code was developed to obtain several relevant plasma radiative properties such as the monochromatic absorption and emission coefficients, mean and multigroup opacities, source functions, radiative power losses, specific intensities and plasma transmission. A detailed

Table 1

Set of configurations and the numbers of relativistic configurations, levels and line transitions considered in the calculations performed by ABAKO/RAPCAL code for carbon ions $\left(n \leq 10, n^{\prime} \leq 6\right)$.

\begin{tabular}{lllllll}
\hline & C-like & B-like & Be-like & Li-like & He-like & H-like \\
\hline & $(2)^{4}$ & $(2)^{3}$ & $(2)^{2}$ & $(2)^{1}$ & $1 s^{2}$ & $1 s^{1}$ \\
& $(2)^{3} n l$ & $(2)^{2} n l$ & $(2)^{1} n l$ & $1 s^{2} n l$ & $1 s^{1} n l$ & $n l$ \\
& $(2)^{2}(3)^{2}$ & $(2)^{1}(3)^{2}$ & $(3)^{2}$ & $1 s^{1}(2)^{2}$ & $(2)^{2}$ & \\
& $(2)^{2}(3)^{1} n^{\prime} l$ & $(2)^{1}(3)^{1} n^{\prime} l$ & $(3)^{1} n^{\prime} l$ & $1 s^{1}(2)^{1} n^{\prime} l$ & $(2)^{1} n^{\prime} l$ & \\
& & $1 s^{1}(2)^{4}$ & $1 s^{1}(2)^{3}$ & $1 s^{1}(3)^{2}$ & $(3)^{2}$ & \\
Configurations & & $1 s^{1}(2)^{3} n^{\prime} l$ & $1 s^{1}(2)^{2} n^{\prime} l$ & $1 s^{1}(3)^{1} n^{\prime} l$ & $(3)^{1} n^{\prime} l$ & \\
Levels & 1677 & 1299 & 644 & 519 & 352 & 100 \\
Transitions & 16,616 & 8871 & 2789 & 2642 & 910 & 100 \\
\hline
\end{tabular}


description of the code can be found in [22]. For this work, the interest has been mainly focused on the spectrally resolved and mean opacities. The monochromatic absorption coefficient, denoted as $\kappa(v)$, includes the boundbound, bound-free and free-free contributions:

$\kappa(v)=\kappa_{b b}(v)+\kappa_{b f}(v)+\kappa_{f f}(v)$,

where $v$ is the photon frequency. The bound-bound contribution to the absorption coefficient is given by

$\kappa_{b b}(v)=\sum_{\zeta} \sum_{i, j} \kappa_{\zeta i \rightarrow \zeta j}(v)$

with

$\kappa_{\zeta i \rightarrow \zeta j}(v)=\frac{h v}{4 \pi} N_{\zeta i} \frac{g_{\zeta j}}{g_{\zeta i}} \frac{c^{2}}{2 h v_{i j}^{3}} A_{\zeta j \rightarrow \zeta i} \phi_{i j}(v)\left(1-\frac{g_{\zeta i}}{g_{\zeta j}} \frac{N_{\zeta j}}{N_{\zeta i}}\right)$,

where $A_{\zeta j \rightarrow \zeta i}$ is the Einstein coefficient for spontaneous deexcitation [41] between the bound states $j, i$ of the ion $\zeta$, $h$ is the Planck's constant, $c$ is the speed of light and $g_{\zeta i}$ and $g_{b j}$ are the statistical weights of the $i$ and $j$ levels. In the previous equations, $\phi_{i j}(v)$ represents the line profile and in its evaluation natural, Doppler and collisional or electron-impact [42] broadenings were included. The line-shape function is applied with the Voigt profile that incorporates all these broadenings. The natural broadening is given by

$\Gamma_{n a t}=\sum_{m, m<i} A_{\zeta i \rightarrow \zeta m}+\sum_{m, m>j} A_{\zeta j \rightarrow \zeta m}$,

the Doppler broadenings is given by

$\Gamma_{\text {Dop }}=\sqrt{\frac{2 T_{i}}{M c^{2}}} v_{i j}$

where $T_{i}$ is the ionic temperature and $M$ is the ion mass. And, finally, the collisional broadening is given by

$$
\begin{aligned}
\Gamma_{\text {coll }}= & n_{e} \frac{2 h^{2}}{3 m_{e}^{2}}\left(\frac{2 m_{e}}{\pi T}\right)^{1 / 2} \frac{\pi}{\sqrt{3}}\left(0.9-\frac{1.1}{\zeta+1}\right) \sum_{\mu}\left[\frac{3 n_{\mu}}{2(\zeta+1)}\right]^{2} \\
& \left(n_{\mu}^{2}-l_{\mu}^{2}-l_{\mu}-1\right),
\end{aligned}
$$

where $n_{e}$ is the electron density, $m_{e}$ is the electron mass, $\mu$ running over the relativistic orbitals involved in the transition, and finally, $n$ and $l$ are the principal and angular quantum number of the orbitals, respectively. by

The bound-free contribution to the absorption is given

$\kappa_{b f}(v)=\sum_{\zeta, i} \sum_{\zeta, j} \kappa_{\zeta i \rightarrow \zeta+1 j}(v)$,

with

$\kappa_{\zeta i \rightarrow \zeta+1 j}(v)=N_{\zeta i} \sigma_{\zeta i \rightarrow \zeta+1 j}^{p h o}(v)\left(1-\frac{N_{\zeta+1 j} n_{e} f(\varepsilon)}{N_{\zeta i}} \frac{g_{\zeta i}}{g_{\zeta+1 j} g(\varepsilon)}\right)$,

where $f(\varepsilon)$ is the Maxwell-Boltzmann distribution for the free electrons and $g(\varepsilon)$ is the density of states with free electron energy $\varepsilon$ which, assuming an ideal gas of free electrons, is given by

$g(\varepsilon)=4 \pi\left(\frac{2 m_{e}}{h^{2}}\right)^{3 / 2} \varepsilon^{1 / 2}$

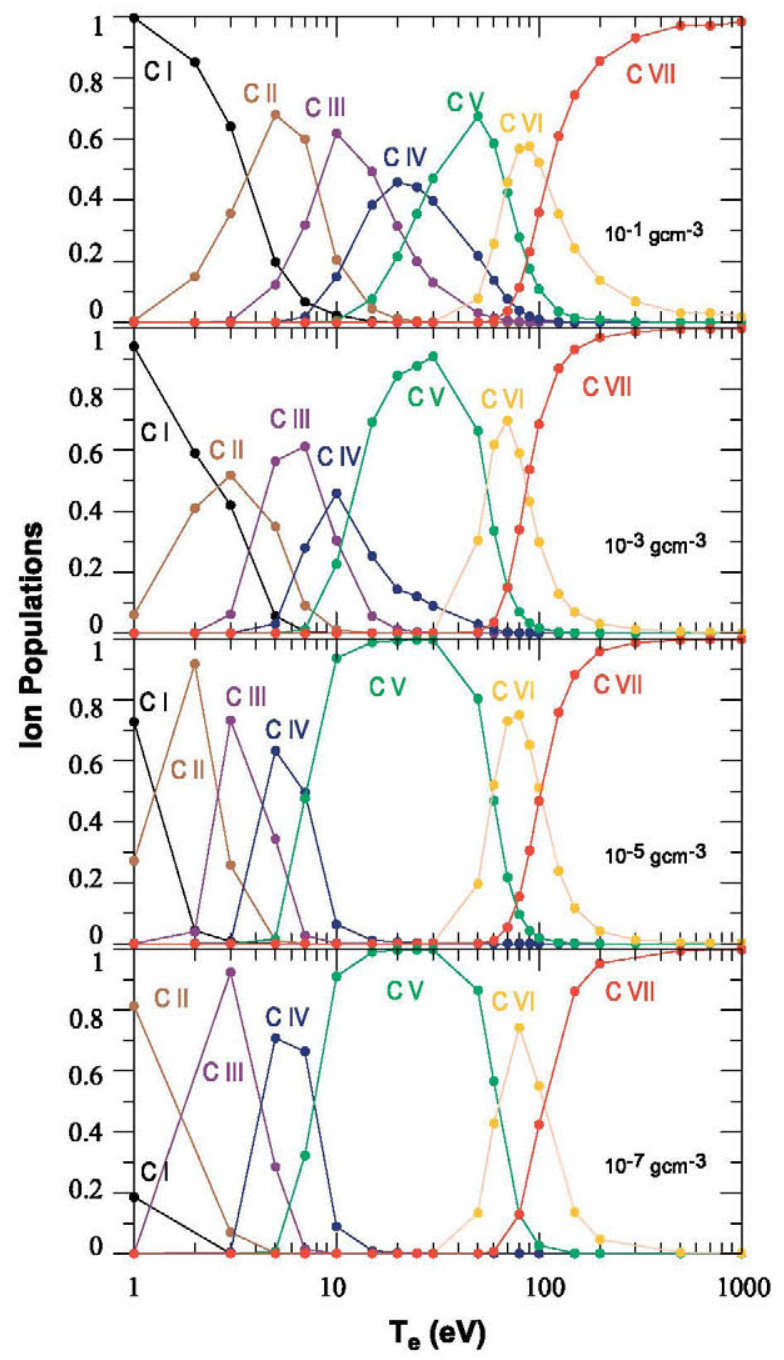

Fig. 1. Fractional carbon ion populations for several matter densities vs. the electron temperature.

The photoionization cross section, $\sigma_{\zeta i \rightarrow \zeta+1 j}^{p h o}(v)$, has been evaluated using the semiclassical expression of Kramers [35]. Finally, the free-free contribution to the absorption coefficient is given by

$\kappa_{f f}(v)=\sum_{\zeta} N_{\zeta} \sigma_{\zeta}^{i b r}(v)\left(1-e^{-h v / T_{e}}\right)$,

where a Maxwell-Boltzmann distribution for the free electrons has been assumed. The Kramers semi-classical expression for the inverse bremsstrahlung cross section of each ion state $\zeta$ has been used [44]

$\sigma_{\zeta}^{i b r}(v)=\frac{16 \pi^{2} e^{2} h^{2} \alpha}{3 \sqrt{3}\left(2 \pi m_{e}\right)^{3 / 2}} \frac{\zeta^{2} n_{e}}{T_{e}^{1 / 2}(h v)^{3}}$,

where $\alpha$ is the fine structure constant. Finally, it is obtained

$\kappa_{f f}(v)=\frac{16 \pi^{2} e^{2} h^{2} \alpha}{3 \sqrt{3}\left(2 \pi m_{e}\right)^{3 / 2}} \frac{\overline{Z^{2}} n_{i o n} n_{e}}{T_{e}^{1 / 2}(h v)^{3}}\left(1-e^{-h v / T_{e}}\right)$, 
with $n_{\text {ion }}$ the total ion density and alpha the fine structure constant. In order to determine the opacity, $k(v)$, it is also taken into account the absorption due to the scattering of photons. In RAPCAL this one is approximated using the Thomson scattering cross section [43]

$\kappa_{\text {scatt }}=n_{e} \sigma^{\text {Thom }}$,

with $\sigma^{\text {Thom }}=6.65 \times 10^{-25} \mathrm{~cm}^{2}$.

Finally, the opacity is given by

$k(v)=\frac{1}{\rho}\left(\kappa(v)+\kappa_{s c a t t}\right)$,

with $\rho$ the matter density.

As it was said in Introduction, in the resolution of the radiative transport equation it is necessary to define the multigroup or mean opacities as the average of the spectrally resolved opacity by using a convenient distribution function of the photon frequency. If we select a huge amount of multigroups of frequency, such as the opacity can be considered nearly constant function of the frequency into each group, then the choice of distribution function is not crucial. In the other case, the use of different weighting functions can lead to quite different results. In practice, the mean opacity is generally taken as either a group Planck and group Rosseland mean opacities which are given by [8]

$k_{P}\left(v_{i}, v_{i+1}\right)=\int_{v_{i}}^{v_{i+1}} d v \tilde{B}(v, T)\left(k(v)-\kappa_{\text {scatt }} / \rho\right)$

$\frac{1}{k_{R}\left(v_{i}, v_{i+1}\right)}=\int_{v_{i}}^{v_{i+1}} d v \frac{\partial \tilde{B}(v, T)}{\partial T} \frac{1}{k(v)}$,

where $\tilde{B}(v, T)$ is the normalized Planckian function,

$\tilde{B}(v, T)=\frac{15}{\pi^{4} T} \frac{u^{3}}{e^{u}-1}, \quad u=\frac{h v}{T}$,

where the interval $\left(v_{i}, v_{i+1}\right)$ defines each frequency group. At the grey transport approximation only one group is considered, and, in this case, the interval of frequencies is $(0, \infty)$.

\section{Results}

This section is divided into three parts. The first one is devoted to the analysis of the average ionization, the ion populations and the thermodynamic regimes as a function of the plasma conditions. This study will allow us to identify which are the most abundant ions that contribute to the radiative opacity of the optically thin carbon plasmas and also when LTE or NLTE regimes can be assumed. In the second part it is carried out an study of the influence of the thermodynamic regime into the spectrally resolved and mean opacities, by means of the comparison between the opacities calculated using the CRSS and the SB equations implemented in ABAKO. Finally, in the third part, it is performed a similar analysis on the multigroup opacities. The ranges of electron temperatures and matter densities considered in this work are $1-10^{3} \mathrm{eV}$ and $10^{-12}-10^{-1} \mathrm{~g} \mathrm{~cm}^{-3}$, respectively. These plasma conditions provide electron densities in the range $10^{10}-10^{22} \mathrm{~cm}^{-3}$.
Table 2

Average ionization for optically thin carbon plasmas in a wide range of plasma conditions. $T_{e}$ in $\mathrm{eV}$ and $\rho$ in $\mathrm{g} \mathrm{cm}^{-3}$

\begin{tabular}{|c|c|c|c|c|c|c|}
\hline$T_{e} / \rho$ & $10^{-12}$ & $10^{-11}$ & $10^{-10}$ & $10^{-9}$ & $10^{-8}$ & $10^{-7}$ \\
\hline 1 & 0.673 & 0.688 & 0.740 & 0.827 & 0.865 & 0.814 \\
\hline 3 & 1.319 & 1.344 & 1.428 & 1.640 & 1.844 & 1.934 \\
\hline 5 & 1.960 & 1.990 & 2.022 & 2.051 & 2.219 & 2.721 \\
\hline 7 & 2.597 & 2.659 & 2.685 & 2.743 & 2.989 & 3.306 \\
\hline 10 & 3.691 & 3.693 & 3.696 & 3.706 & 3.773 & 3.911 \\
\hline 15 & 3.973 & 3.973 & 3.973 & 3.976 & 3.980 & 3.992 \\
\hline 20 & 3.993 & 3.993 & 3.993 & 3.996 & 3.995 & 3.998 \\
\hline 25 & 3.997 & 3.997 & 3.997 & 3.997 & 3.998 & 3.999 \\
\hline 30 & 3.999 & 3.999 & 3.999 & 3.999 & 3.999 & 3.999 \\
\hline 50 & 4.097 & 4.097 & 4.098 & 4.098 & 4.104 & 4.134 \\
\hline 60 & 4.343 & 4.343 & 4.344 & 4.347 & 4.367 & 4.441 \\
\hline 80 & 4.935 & 4.935 & 4.935 & 4.938 & 4.958 & 5.000 \\
\hline 100 & 5.375 & 5.375 & 5.375 & 5.376 & 5.384 & 5.396 \\
\hline 150 & 5.859 & 5.859 & 5.859 & 5.859 & 5.860 & 5.861 \\
\hline 200 & 5.951 & 5.951 & 5.951 & 5.951 & 5.951 & 5.952 \\
\hline 500 & 5.996 & 5.996 & 5.999 & 5.996 & 5.996 & 5.996 \\
\hline 1000 & 5.999 & 5.999 & 5.999 & 5.999 & 5.999 & 5.999 \\
\hline$T_{e} / \rho$ & $10^{-6}$ & $10^{-5}$ & $10^{-4}$ & $10^{-3}$ & $10^{-2}$ & $10^{-1}$ \\
\hline 1 & 0.575 & 0.272 & 0.133 & 0.060 & 0.018 & 0.005 \\
\hline 3 & 1.929 & 1.731 & 1.198 & 0.642 & 0.581 & 0.364 \\
\hline 5 & 2.854 & 2.654 & 2.084 & 1.569 & 1.197 & 0.923 \\
\hline 7 & 3.568 & 3.450 & 2.886 & 2.201 & 1.686 & 1.285 \\
\hline 10 & 3.962 & 3.936 & 3.684 & 2.899 & 2.278 & 1.908 \\
\hline 15 & 3.996 & 3.989 & 3.925 & 3.635 & 3.025 & 2.486 \\
\hline 20 & 3.999 & 3.995 & 3.961 & 3.831 & 3.433 & 2.873 \\
\hline 25 & 3.999 & 3.997 & 3.975 & 3.872 & 3.623 & 3.146 \\
\hline 30 & 4.000 & 3.999 & 3.983 & 3.908 & 3.730 & 3.338 \\
\hline 50 & 4.165 & 4.196 & 4.254 & 4.281 & 4.226 & 3.799 \\
\hline 60 & 4.490 & 4.540 & 4.631 & 4.678 & 4.611 & 4.102 \\
\hline 80 & 5.020 & 5.058 & 5.168 & 5.268 & 5.190 & 4.750 \\
\hline 100 & 5.407 & 5.450 & 5.576 & 5.669 & 5.602 & 5.230 \\
\hline 150 & 5.866 & 5.882 & 5.918 & 5.929 & 5.896 & 5.728 \\
\hline 200 & 5.954 & 5.959 & 5.971 & 5.969 & 5.949 & 5.845 \\
\hline 500 & 5.996 & 5.997 & 5.997 & 5.995 & 5.980 & 5.969 \\
\hline 1000 & 5.999 & 5.999 & 5.999 & 5.998 & 5.991 & 5.982 \\
\hline
\end{tabular}

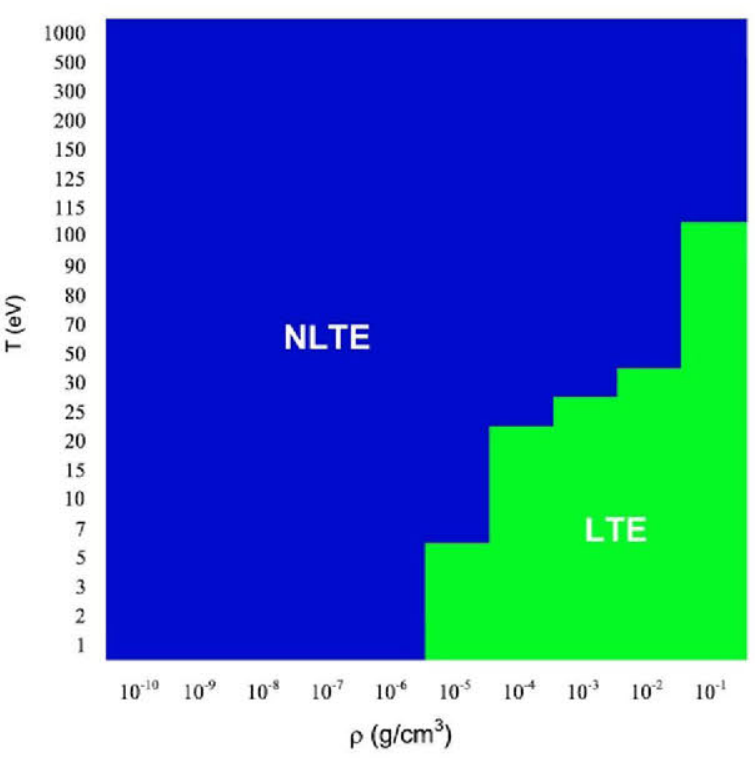

Fig. 2. Thermodynamical regimes of carbon plasmas as a function of the density of matter and electron temperature. 


\subsection{Analysis of the ion populations and thermodynamic} regimes

As it was said in Introduction, the knowledge of the thermodynamic regimes and the ion populations is a valuable information for further calculations. By means of the resolution of the CRSS implemented in ABAKO we have obtained the average ionization and the fractional ion populations in the range of temperature and density before mentioned. Thus, in Fig. 1 are shown the fractional ion populations of the seven ions of carbon and in Table 2 are listed the values of the average ionization for several plasma conditions. From the figure we can distinguish three regions in the electron temperature. The first one is associated to temperatures between 1 and $10 \mathrm{eV}$. Only in this range of temperatures the fractional populations of $\mathrm{C}$ like and B-like ions are noticeable. Their relative relevance decreases as the temperature grows up. Moreover, it is also observed that the relevance of $\mathrm{C}$-like ion decreases as the density of matter does. As it was concluded in $[39,49]$, when the less ionized ions of carbon are the most abundant, i.e. in this range of plasma conditions, accurate

Table 3

Planck and Rosseland mean opacities $\left(\mathrm{cm}^{2} \mathrm{~g}^{-1}\right)$ for carbon plasmas calculated from ABAKO and LEDCOP codes assuming LTE regime.

\begin{tabular}{|c|c|c|c|c|c|c|c|c|}
\hline \multicolumn{9}{|c|}{$\rho\left(\mathrm{g} \mathrm{cm}^{-3}\right)$} \\
\hline \multirow[t]{3}{*}{$T_{e}(\mathrm{eV})$} & \multicolumn{4}{|l|}{$10^{-1}$} & \multicolumn{4}{|l|}{$10^{-3}$} \\
\hline & \multicolumn{2}{|l|}{$\kappa_{P}$} & \multicolumn{2}{|l|}{$\kappa_{R}$} & \multicolumn{2}{|l|}{$\kappa_{P}$} & \multicolumn{2}{|l|}{$\kappa_{R}$} \\
\hline & ABAKO & LEDCOP & ABAKO & LEDCOP & ABAKO & LEDCOP & ABAKO & LEDCOP \\
\hline 1 & $1.08 \times 10^{5}$ & $9.74 \times 10^{4}$ & $4.09 \times 10^{4}$ & $8.820 \times 10^{3}$ & $8.62 \times 10^{4}$ & $8.60 \times 10^{4}$ & $1.09 \times 10^{4}$ & $1.10 \times 10^{4}$ \\
\hline 10 & $1.34 \times 10^{5}$ & $1.69 \times 10^{5}$ & $5.36 \times 10^{4}$ & $6.90 \times 10^{4}$ & $4.78 \times 10^{4}$ & $5.14 \times 10^{4}$ & $2.52 \times 10^{4}$ & $1.80 \times 10^{4}$ \\
\hline 30 & $7.46 \times 10^{3}$ & $9.63 \times 10^{3}$ & $9.62 \times 10^{2}$ & $1.80 \times 10^{3}$ & $1.04 \times 10^{3}$ & $5.58 \times 10^{2}$ & $4.00 \times 10^{1}$ & $4.40 \times 10^{1}$ \\
\hline 50 & $9.15 \times 10^{3}$ & $9.00 \times 10^{3}$ & $6.83 \times 10^{3}$ & $8.50 \times 10^{3}$ & $2.22 \times 10^{3}$ & $2.65 \times 10^{3}$ & $2.60 \times 10^{1}$ & $2.60 \times 10^{1}$ \\
\hline 100 & $3.98 \times 10^{3}$ & $3.01 \times 10^{3}$ & $7.17 \times 10^{2}$ & $7.94 \times 10^{2}$ & $9.60 \times 10^{1}$ & $4.00 \times 10^{1}$ & $0.50 \times 10^{1}$ & $0.60 \times 10^{1}$ \\
\hline 200 & $1.82 \times 10^{2}$ & $1.55 \times 10^{2}$ & $2.70 \times 10^{1}$ & $2.60 \times 10^{1}$ & $0.21 \times 10^{1}$ & $0.14 \times 10^{1}$ & $6.50 \times 10^{1}$ & $6.50 \times 10^{1}$ \\
\hline
\end{tabular}

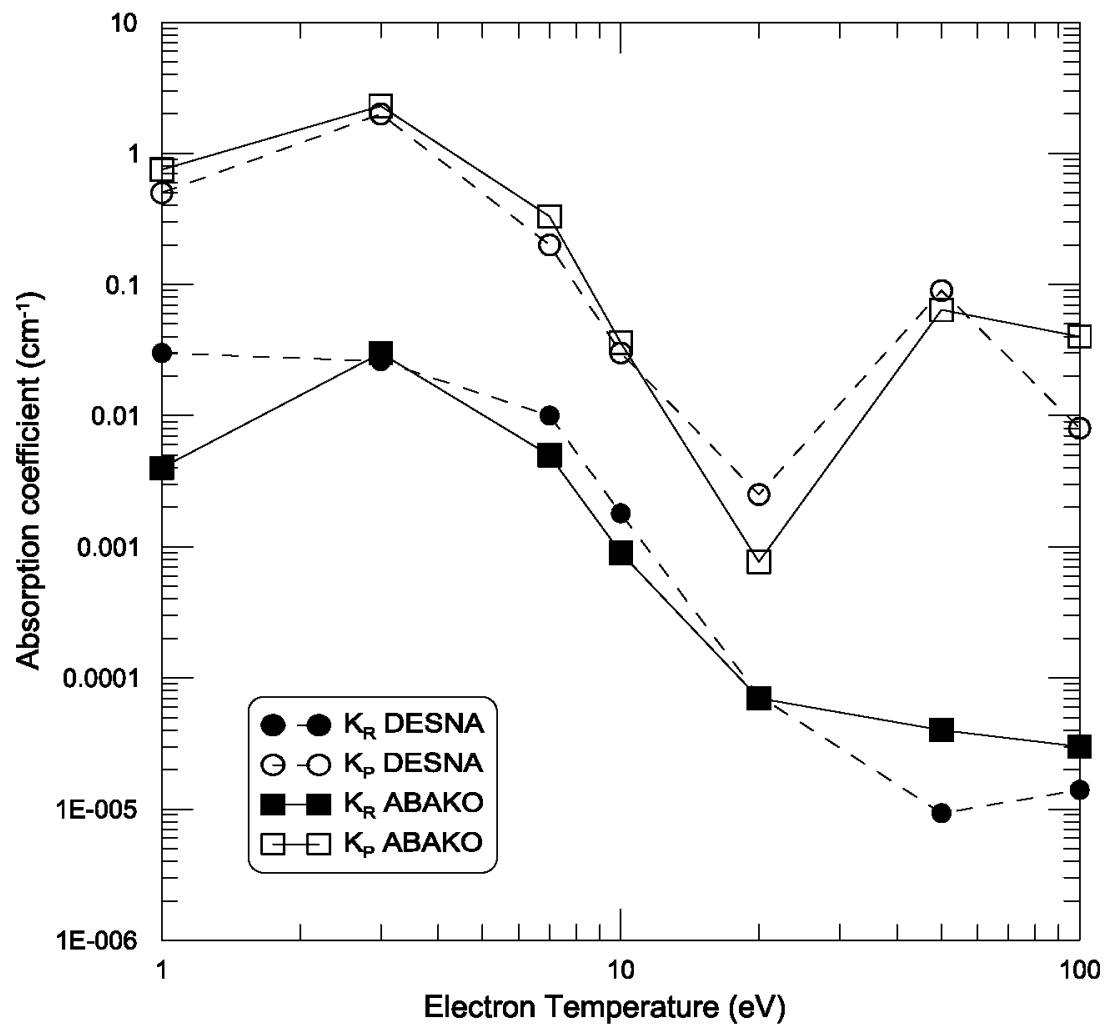

Fig. 3. Comparison between ABAKO and DESNA results of the Planck and Rosseland mean absorption coefficients vs. the electron temperature. The density of matter is $10^{-5} \mathrm{~g} \mathrm{~cm}^{-3}$. 
calculations of plasma atomic level populations and radiative properties require that the atomic data are obtained under the DLA approach including configuration-interaction effects. The range of intermediate temperatures $(10-50 \mathrm{eV})$ constitutes the second region. In this case and for all the densities considered, the fractional populations of the neutral carbon and the fully stripped ion are not noticeable. For densities higher than $10^{-4} \mathrm{~g} \mathrm{~cm}^{-3}$ there are contributions from B-like to $\mathrm{H}$-like ions. On the other hand, for lower densities we find that He-like is the most abundant ion with fractional populations always larger than 0.8 being almost the only ion with relevant abundance for temperatures between 15 and $40 \mathrm{eV}$. Finally, for the range of temperatures $25-50 \mathrm{eV}$
He-like and $\mathrm{H}$-like ions are the only ones present in the plasma. Thus, for example, their fractional population at $50 \mathrm{eV}$ and $10^{-5} \mathrm{~g} \mathrm{~cm}^{-3}$ are 0.80 and 0.20 , respectively, or 0.85 and 0.15 , respectively, at $50 \mathrm{eV}$ and $10^{-7} \mathrm{~g} \mathrm{~cm}^{-3}$. Furthermore, as it was concluded in [27] for matter densities lower than $10^{-7} \mathrm{~g} \mathrm{~cm}^{-3}$ and temperatures between 25 and $50 \mathrm{eV}$ the carbon plasma can be assumed as in $\mathrm{CE}$ and, therefore, the must populated atomic levels must be the ground ones which implies that the spectrally resolved line opacity must be mainly ruled by the $\mathrm{He}_{\alpha, \beta, \gamma}$ lines and, in minor proportion, by the $\mathrm{H}_{\alpha, \beta, \gamma}$ ones. Finally, the third region is associated to temperatures ranged from 50 to $1000 \mathrm{eV}$. In this case, the ions present in the plasma are Li-like, He-like, H-like and the fully stripped
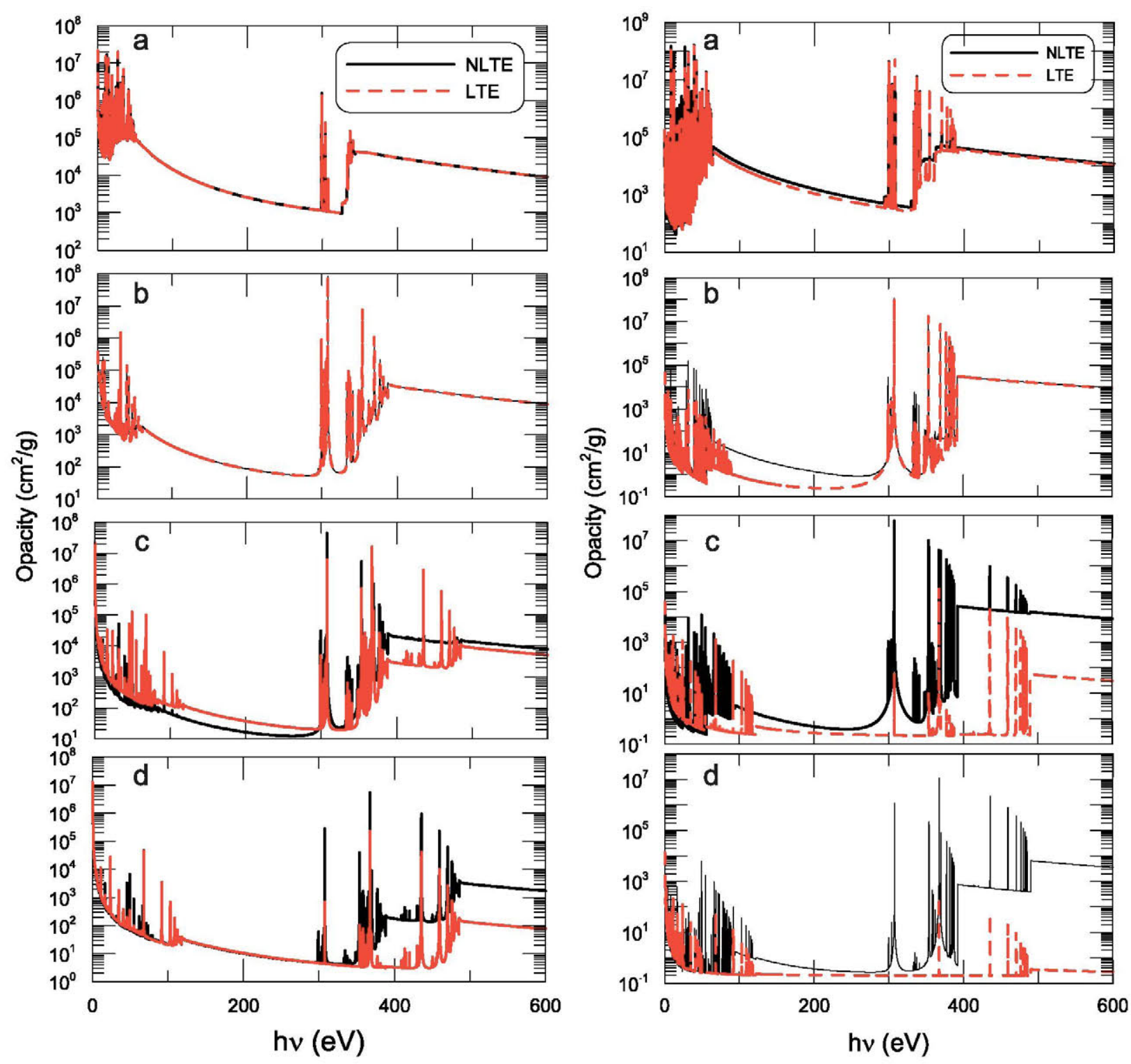

Fig. 4. Spectrally resolved opacity calculated under NLTE and LTE thermodynamic regimes. The density of matter is $10^{-3} \mathrm{~g} \mathrm{~cm}^{-3} \mathrm{on}$ the left column and $10^{-6} \mathrm{~g} \mathrm{~cm}^{-3}$ on the right one. The electron temperatures are (a) 5, (b) 20 , (c) 50 and (d) $100 \mathrm{eV}$. 
one, disappearing the Li-like ion for densities lower than $10^{-5} \mathrm{~g} \mathrm{~cm}^{-3}$ and for higher densities for temperatures larger than $100 \mathrm{eV}$. For this last density of matter, and for lower ones, and at a temperature of $63 \mathrm{eV}$ we have found that only the He-like and H-like ions are present in the plasma and both with the same fractional population, i.e. 0.50 . The contribution of the fully stripped ion becomes noticeable for temperatures higher than this. We can see form the figure that the maximum value of the fractional population of $\mathrm{H}$-like ion is reached at temperatures around $80 \mathrm{eV}$ for all the densities considered, decreasing when the temperature increases. Finally, for temperatures higher than $100 \mathrm{eV}$ the $\mathrm{H}$-like and the fully stripped ions are almost the only ones present in the plasma and the later becomes the most relevant ion for temperatures higher than $115 \mathrm{eV}$. In this case the line opacity is mainly ruled by the $\mathrm{H}$-like ion lines and when the $\mathrm{CE}$ can be assumed the $\mathrm{H}_{\alpha, \beta, \gamma}$ lines are, practically, the only contributions to the line opacity.

Obviously, the wide range of plasma conditions considered in this work covers different thermodynamic regimes, i.e. LTE or NLTE. The knowledge of the plasma regime for a given density and temperature condition could entail a considerable saving in calculation time since the resolution of SB equations is considerably faster than CRM ones and the complexity is also reduced. Therefore, it is very useful to have criteria that permit us to establish the thermodynamic regime of the plasma for given electron temperature and density of matter. In the literature, there are available some qualitative criteria to estimate when an ion or ion level can be considered under LTE conditions [45,46]. However, in this work it is employed a criterion that can state the regime of the whole plasma: when the ion populations, $p_{i}$, calculated from $\mathrm{SB}$ equations, $p_{i}^{S B}$, present a mean deviation $(\Delta p)$, with respect to those obtained from the CRSS model, $p_{i}^{\text {CRSS }}$, smaller than a certain value imposed $\left(\Delta p^{*}\right)$ then it is considered that LTE regime has been achieved. Otherwise, the plasma is under NLTE conditions. The mean deviation is calculated as

$\Delta p^{L T E}=\sqrt{\frac{\sum_{i}\left(p_{i}^{C R S S}-p_{i}^{S B}\right)^{2}}{\sum_{i}\left(p_{i}^{S B}\right)^{2}}} \leq \Delta p^{*}$,

where $i$ runs over the whole set of ions. This criterion was applied to carbon [27], aluminum [22] and xenon [47,48] plasmas. In those works the maps of the thermodynamic regimes were obtained by fixing $\Delta p^{*}=0.1(=10 \%)$. It was proved in [48] that when this criterion is fulfilled for the whole plasma the Griems criterion for each ion in the plasma is, in general, also verified. This fact is expected since the two conditions that must be fulfilled in Griem's criterion can be also obtained using the Lotz and Kramers formulas for the ionization and radiative recombination rate coefficients [46], which are the ones used in ABAKO for that processes. In Fig. 2 it is shown the map of thermodynamic regimes for an optically thin carbon plasma according to the criterion of Eq. (19). It is observed that as the density decreases the maximum value of the temperature at which the plasma can be assumed under LTE conditions also does. Furthermore, we obtained that for matter densities less or equal to $10^{-6} \mathrm{~g} \mathrm{~cm}^{-3}$ the mean relative differences between LTE and NLTE ion populations are always larger than $10 \%$ and, therefore, according to the criterion purposed, the plasma is in NLTE. We have also checked that the fulfilment of this criterion in the ion populations also ensures a good agreement between atomic level populations, and therefore in the radiative properties, calculated using the CRSS or the SB equations, obtaining relative differences around or lower than $10 \%$ in the Rosseland and Planck mean opacities, as it is shown in the next subsection.

For plasma temperatures lower than $100 \mathrm{eV}$ we have obtained that this criterion ensured that the relative deviations in the average ionization, $\Delta \bar{Z}$, were always around or lower than $1 \%$ and therefore the criterion of

Table 4

Analysis of the LTE criterion (in percentage) for Rosseland and Planck mean opacities for several temperatures and matter densities. $T_{e}$ and $\rho$ are given in $\mathrm{eV}$ and in $\mathrm{g} \mathrm{cm}^{-3}$, respectively.

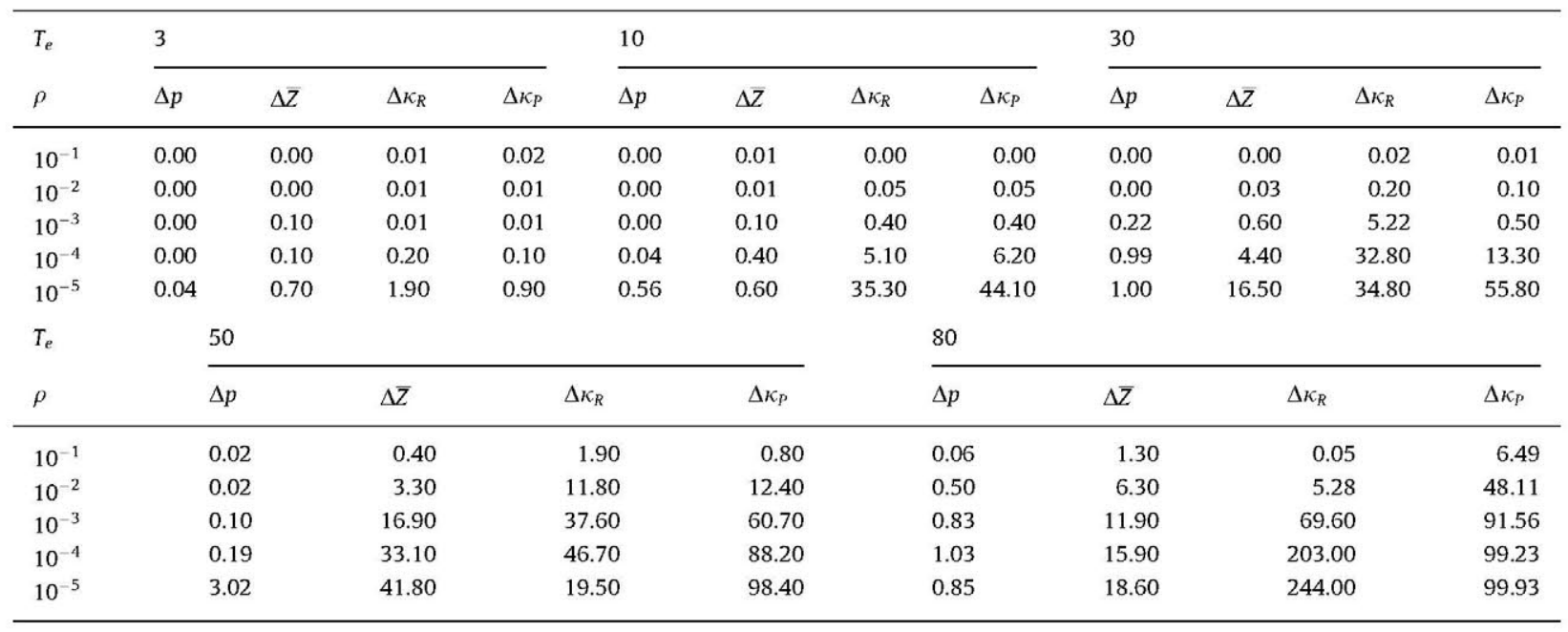


Eq. (19) can be substituted by a criterion of a $1 \%$ on the average ionization. However, for electron temperatures higher than $100 \mathrm{eV}$ and densities larger than $10^{-5} \mathrm{~g} \mathrm{~cm}^{-3}$ this criterion based on the average ionization is no longer valid, since we have obtained values of $\Delta \bar{Z}$ lower than $1 \%$ whereas the criterion in ion populations is not fulfilled and, therefore, the plasma is in NLTE, see Fig. 2. Furthermore, it is worth pointing out that for these plasma conditions, where only the $\mathrm{H}$-like and the fully stripped ions are present in the plasma, the criterion of Eq. (19) must be applied without including the fully stripped ion in the calculation of the mean relative deviation in order to ensure that $\Delta p$ is greater than 0.1 . For temperatures higher than $200 \mathrm{eV}$ the fractional population of the $\mathrm{H}$-like ion becomes insignificant and therefore the criterion would predict that the plasma is in LTE. However, as it is known, for a given density, as the temperature increases the NLTE regime must remain, as we obtain when we compare the H-like level populations and the mean opacities. Finally, we would like to point out that as
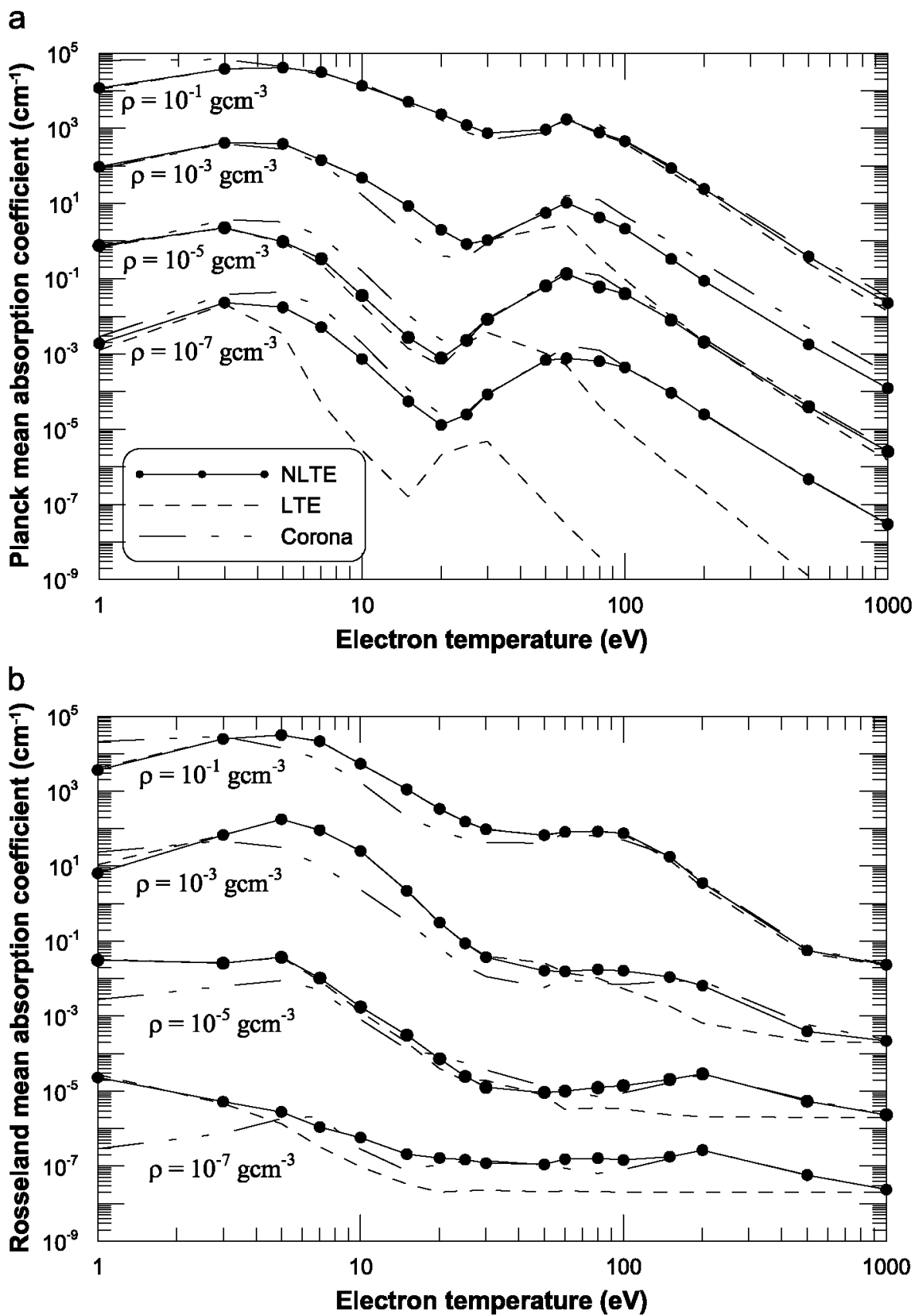

Fig. 5. Comparison of NLTE and LTE Planck (a) and Rosseland (b) mean absorption coefficients vs. electron temperature for several densities of matter. 
we have assumed in our CRSS model a Maxwell-Boltzmann distribution for the free electrons (as many available collisional-radiative codes), at very high temperatures CRSS and SB calculations of the radiative and mean opacities will be very similar since the main contribution to the opacity is the free-free one.

\subsection{Analysis of the spectrally resolved and mean radiative opacities}

In order to check the results of opacity we show in Table 3 the comparison between the results provided by ABAKO/RAPCAL and LEDCOP codes [50] of the Rosseland and Planck mean opacities. The atomic level populations in both codes have been obtained assuming the plasma in LTE. Generally, it is observed a good qualitative agreement between them and the main differences could be mainly due to the different atomic models assumed in both codes. Thus, in ABAKO it is used a DLA relativistic approach whereas in LEDCOP a detailed-term-accounting (DTA) atomic model based on LS coupling is employed.

We have also performed a comparison of the mean opacities with those provided by DESNA code [51], see Fig. 3 for a density of matter of $10^{-5} \mathrm{~g} \mathrm{~cm}^{-3}$ and for a wide range of electron temperatures. In this case, the mean opacities were calculated in both codes using a CRSS model. Generally, a good agreement is found between both simulations being the main differences due to the atomic model employed since in DESNA is a detailed-average-accounting (DCA) one that includes 600 atomic configurations whereas in ABAKO a DLA model was implemented including about 4500 atomic configurations.

Once it has been checked the qualitative accuracy of the mean opacities provided by ABAKO/RAPCAL, we will analyze the CRSS and SB calculations of the spectrally resolved and mean opacities using this code. Thus, in Fig. 4 are plotted the spectrally resolved opacities for the isodense sequences of $10^{-3} \mathrm{~g} \mathrm{~cm}^{-3}$ and $10^{-6} \mathrm{~g} \mathrm{~cm}^{-3}$ and four temperatures $(5,20,500$ and $100 \mathrm{eV})$ calculated using the same set of atomic configurations but with atomic level populations obtained from both the CRSS and the SB equations. In the figures, each region of the photon energy mesh in which the line transitions are located, i.e. $0-150 \mathrm{eV}$ and $250-600 \mathrm{eV}$, has been divided uniformly into $2 \times 10^{3}$ points. We would like to point out that the number of line transitions included depends on the plasma conditions, due to the effect of the continuum lowering at high densities, and also on the criterion used to skip weak line transitions since we only include

Table 5

Planck mean opacities $\left(\mathrm{cm}^{2} \mathrm{~g}^{-1}\right)$ in a wide range of plasma conditions. $T_{e}$ in $\mathrm{eV}$ and $\rho$ in $\mathrm{g} \mathrm{cm}^{-3}$.

\begin{tabular}{|c|c|c|c|c|c|c|}
\hline$T_{e} / \rho$ & $10^{-12}$ & $10^{-11}$ & $10^{-10}$ & $10^{-9}$ & $10^{-8}$ & $10^{-7}$ \\
\hline 1 & $3.36 \times 10^{4}$ & $3.20 \times 10^{4}$ & $2.72 \times 10^{4}$ & $1.94 \times 10^{4}$ & $1.56 \times 10^{4}$ & $1.92 \times 10^{4}$ \\
\hline 3 & $6.08 \times 10^{5}$ & $5.88 \times 10^{4}$ & $5.25 \times 10^{4}$ & $5.04 \times 10^{4}$ & $4.48 \times 10^{5}$ & $2.29 \times 10^{4}$ \\
\hline 5 & $1.51 \times 10^{5}$ & $1.76 \times 10^{5}$ & $1.93 \times 10^{5}$ & $1.68 \times 10^{5}$ & $2.07 \times 10^{5}$ & $1.73 \times 10^{5}$ \\
\hline 7 & $1.65 \times 10^{5}$ & $1.37 \times 10^{5}$ & $1.29 \times 10^{5}$ & $1.22 \times 10^{5}$ & $8.40 \times 10^{4}$ & $5.17 \times 10^{4}$ \\
\hline 10 & $1.57 \times 10^{4}$ & $1.47 \times 10^{4}$ & $1.45 \times 10^{4}$ & $1.43 \times 10^{4}$ & $1.39 \times 10^{4}$ & $7.28 \times 10^{3}$ \\
\hline 15 & $1.11 \times 10^{3}$ & $1.11 \times 10^{3}$ & $1.10 \times 10^{3}$ & $1.11 \times 10^{3}$ & $1.06 \times 10^{3}$ & $5.47 \times 10^{2}$ \\
\hline 20 & $2.41 \times 10^{2}$ & $2.41 \times 10^{2}$ & $2.41 \times 10^{2}$ & $2.40 \times 10^{2}$ & $2.22 \times 10^{2}$ & $1.29 \times 10^{2}$ \\
\hline 25 & $2.82 \times 10^{2}$ & $2.82 \times 10^{2}$ & $2.82 \times 10^{2}$ & $2.81 \times 10^{2}$ & $2.73 \times 10^{2}$ & $2.46 \times 10^{2}$ \\
\hline 30 & $8.56 \times 10^{2}$ & $8.56 \times 10^{2}$ & $8.55 \times 10^{2}$ & $8.55 \times 10^{2}$ & $8.51 \times 10^{2}$ & $8.40 \times 10^{2}$ \\
\hline 50 & $6.96 \times 10^{3}$ & $6.96 \times 10^{3}$ & $6.95 \times 10^{3}$ & $6.95 \times 10^{3}$ & $6.92 \times 10^{3}$ & $6.76 \times 10^{3}$ \\
\hline 60 & $8.36 \times 10^{3}$ & $8.36 \times 10^{3}$ & $8.36 \times 10^{3}$ & $8.34 \times 10^{3}$ & $8.20 \times 10^{3}$ & $7.68 \times 10^{3}$ \\
\hline 80 & $6.90 \times 10^{3}$ & $6.90 \times 10^{3}$ & $6.90 \times 10^{3}$ & $6.87 \times 10^{3}$ & $6.72 \times 10^{3}$ & $6.40 \times 10^{3}$ \\
\hline 100 & $4.52 \times 10^{3}$ & $4.52 \times 10^{3}$ & $4.52 \times 10^{3}$ & $4.51 \times 10^{3}$ & $4.46 \times 10^{3}$ & $4.38 \times 10^{3}$ \\
\hline 150 & $9.41 \times 10^{2}$ & $9.41 \times 10^{2}$ & $9.41 \times 10^{2}$ & $9.40 \times 10^{2}$ & $9.34 \times 10^{2}$ & $9.25 \times 10^{2}$ \\
\hline 200 & $2.51 \times 10^{2}$ & $2.51 \times 10^{2}$ & $2.51 \times 10^{2}$ & $2.50 \times 10^{2}$ & $2.49 \times 10^{2}$ & $2.46 \times 10^{2}$ \\
\hline 500 & $0.47 \times 10^{1}$ & $0.47 \times 10^{1}$ & $0.47 \times 10^{1}$ & $0.47 \times 10^{1}$ & $0.47 \times 10^{1}$ & $0.46 \times 10^{1}$ \\
\hline 1000 & $3.00 \times 10^{1}$ & $3.00 \times 10^{1}$ & $3.00 \times 10^{1}$ & $3.00 \times 10^{1}$ & $3.00 \times 10^{1}$ & $2.90 \times 10^{1}$ \\
\hline$T_{e} / \rho$ & $10^{-6}$ & $10^{-5}$ & $10^{-4}$ & $10^{-3}$ & $10^{-2}$ & $10^{-1}$ \\
\hline 1 & $4.31 \times 10^{4}$ & $7.54 \times 10^{4}$ & $8.75 \times 10^{4}$ & $9.38 \times 10^{4}$ & $1.00 \times 10^{5}$ & $1.17 \times 10^{5}$ \\
\hline 3 & $2.30 \times 10^{5}$ & $2.26 \times 10^{5}$ & $3.22 \times 10^{5}$ & $4.11 \times 10^{5}$ & $4.99 \times 10^{5}$ & $3.79 \times 10^{5}$ \\
\hline 5 & $1.36 \times 10^{5}$ & $9.67 \times 10^{4}$ & $1.62 \times 10^{5}$ & $3.85 \times 10^{5}$ & $5.51 \times 10^{5}$ & $4.09 \times 10^{5}$ \\
\hline 7 & $3.01 \times 10^{4}$ & $3.37 \times 10^{4}$ & $6.75 \times 10^{4}$ & $1.42 \times 10^{5}$ & $3.56 \times 10^{4}$ & $3.10 \times 10^{5}$ \\
\hline 10 & $2.92 \times 10^{3}$ & $3.55 \times 10^{3}$ & $1.50 \times 10^{4}$ & $4.80 \times 10^{4}$ & $1.59 \times 10^{5}$ & $1.34 \times 10^{5}$ \\
\hline 15 & $2.22 \times 10^{2}$ & $2.73 \times 10^{2}$ & $1.35 \times 10^{3}$ & $8.55 \times 10^{3}$ & $4.00 \times 10^{4}$ & $5.07 \times 10^{4}$ \\
\hline 20 & $6.70 \times 10^{2}$ & $7.70 \times 10^{2}$ & $2.81 \times 10^{2}$ & $1.99 \times 10^{3}$ & $1.11 \times 10^{4}$ & $2.34 \times 10^{4}$ \\
\hline 25 & $2.27 \times 10^{2}$ & $2.30 \times 10^{2}$ & $2.89 \times 10^{2}$ & $8.38 \times 10^{2}$ & $4.43 \times 10^{3}$ & $1.20 \times 10^{4}$ \\
\hline 30 & $8.33 \times 10^{2}$ & $8.33 \times 10^{2}$ & $8.49 \times 10^{2}$ & $1.05 \times 10^{3}$ & $2.67 \times 10^{3}$ & $7.46 \times 10^{3}$ \\
\hline 50 & $6.59 \times 10^{3}$ & $6.42 \times 10^{3}$ & $6.06 \times 10^{3}$ & $5.65 \times 10^{3}$ & $6.01 \times 10^{3}$ & $9.22 \times 10^{3}$ \\
\hline 60 & $7.33 \times 10^{3}$ & $1.34 \times 10^{4}$ & $1.20 \times 10^{4}$ & $1.04 \times 10^{4}$ & $1.06 \times 10^{4}$ & $1.72 \times 10^{4}$ \\
\hline 80 & $6.25 \times 10^{3}$ & $5.98 \times 10^{3}$ & $5.22 \times 10^{3}$ & $4.26 \times 10^{3}$ & $4.60 \times 10^{3}$ & $7.69 \times 10^{3}$ \\
\hline 100 & $4.30 \times 10^{3}$ & $3.98 \times 10^{3}$ & $3.05 \times 10^{3}$ & $2.13 \times 10^{3}$ & $2.32 \times 10^{3}$ & $4.53 \times 10^{3}$ \\
\hline 150 & $8.95 \times 10^{2}$ & $7.87 \times 10^{2}$ & $5.32 \times 10^{2}$ & $3.34 \times 10^{2}$ & $3.59 \times 10^{2}$ & $8.68 \times 10^{2}$ \\
\hline 200 & $2.37 \times 10^{2}$ & $2.07 \times 10^{2}$ & $1.40 \times 10^{2}$ & $8.80 \times 10^{1}$ & $9.20 \times 10^{1}$ & $2.41 \times 10^{2}$ \\
\hline 500 & $0.44 \times 10^{1}$ & $0.39 \times 10^{1}$ & $0.28 \times 10^{1}$ & $0.18 \times 10^{1}$ & $0.17 \times 10^{1}$ & $0.38 \times 10^{1}$ \\
\hline 1000 & $2.80 \times 10^{1}$ & $2.50 \times 10^{1}$ & $1.90 \times 10^{1}$ & $1.20 \times 10^{1}$ & $1.20 \times 10^{1}$ & $2.50 \times 10^{1}$ \\
\hline
\end{tabular}


transitions from atomic levels with fractional populations larger than $10^{-12}$. For example, for a given density of matter of $10^{-3} \mathrm{~g} \mathrm{~cm}^{-3}$ and electron temperatures of $5 \mathrm{eV}$ and $50 \mathrm{eV}$ the numbers of transitions considered are around $10^{5}$ and $10^{3}$, respectively. With respect to the orders of magnitude of the different line broadenings considered, i.e natural, Doppler and collisional, they depend on plasma conditions. Thus, for example, for a density of $10^{-3} \mathrm{~g} \mathrm{~cm}^{-3}$ and at $5 \mathrm{eV}$, they are around $10^{-5}$, $10^{-4}$ and $1 \mathrm{eV}$, respectively. For the same temperature but for a density of matter of $10^{-6} \mathrm{~g} \mathrm{~cm}^{-3}$ the collisional broadening decreases to $10^{-3} \mathrm{eV}$, whereas for the density of matter of $10^{-3}$ but for an electron temperature of $50 \mathrm{eV}$ the Doppler broadening increases to $10^{-3}$. At the density of matter of $10^{-3} \mathrm{~g} \mathrm{~cm}^{-3}$ and electron temperatures of 5 and $20 \mathrm{eV}$ both spectrally resolved opacities are very similar whereas for the other two temperatures, 50 and $100 \mathrm{eV}$, noticeable discrepancies are observed. These results agree with the ones obtained using the criterion of Eq. (19) (see Fig. 2) since it predicted that for this density the change from LTE to NLTE of the plasma regime is produced for temperatures higher than $30 \mathrm{eV}$. Thus, for example, at $30 \mathrm{eV}$ we obtained that $\Delta p=0.03$ (and $\Delta \bar{Z}=0.60$ ) and relative differences, in percentage, in the Rosseland and Planck mean opacities around $5.20 \%$ and
$0.50 \%$, respectively. On the other hand, for a temperature of $50 \mathrm{eV}, \Delta p=0.88$ and $\Delta \bar{Z}=1.70$, and the relative differences in the Rosseland and Planck mean opacities are, in this case, around 60\%. For the density of matter of $10^{-6} \mathrm{~g} \mathrm{~cm}^{-3}$ the plasma is in NLTE for all the temperatures considered (see Fig. 2) and, therefore, in Fig. 4 we can detect significant differences between CRSS and SB opacities. For example, at $20 \mathrm{eV}$ the relative differences in the Planck and Rosseland mean opacities are $62 \%$ and more than $100 \%$, respectively. More examples of the relative differences, in percentage, between CRSS and SB calculations of the ion populations, average ionizations and in the mean opacities are listed in Table 4. From the table it is detected that when LTE regime can be assumed, according to the criterion in the ion populations, the relative differences in the Rosseland and Planck mean opacities calculated using the CRSS and the SB equations are always around or lower than $10 \%$. As it is known, the mean opacities depend on the weighting function the Planckian distribution of photon energies or its temperature derivative) whereas that dependence is weaker in the calculation of the multigroup opacities (in particular, as the number of groups increase that dependence tends to vanish). As a consequence, in the calculations of the mean opacities, the main contribution comes from those

Table 6

Rosseland mean opacities $\left(\mathrm{cm}^{2} \mathrm{~g}^{-1}\right)$ in a wide range of plasma conditions. $T_{\mathrm{e}}$ in $\mathrm{eV}$ and $\rho$ in $\mathrm{g} \mathrm{cm}^{-3}$.

\begin{tabular}{|c|c|c|c|c|c|c|}
\hline$T_{e} / \rho$ & $10^{-12}$ & $10^{-11}$ & $10^{-10}$ & $10^{-9}$ & $10^{-8}$ & $10^{-7}$ \\
\hline 1 & $1.10 \times 10^{1}$ & $1.10 \times 10^{1}$ & $1.20 \times 10^{1}$ & $1.70 \times 10^{1}$ & $0.20 \times 10^{1}$ & $2.32 \times 10^{2}$ \\
\hline 3 & $5.40 \times 10^{1}$ & $5.80 \times 10^{1}$ & $6.10 \times 10^{1}$ & $7.80 \times 10^{1}$ & $0.29 \times 10^{1}$ & $5.20 \times 10^{1}$ \\
\hline 5 & $7.40 \times 10^{1}$ & $9.70 \times 10^{1}$ & $0.10 \times 10^{1}$ & $0.21 \times 10^{1}$ & $0.40 \times 10^{1}$ & $2.80 \times 10^{1}$ \\
\hline 7 & $7.90 \times 10^{1}$ & $8.30 \times 10^{1}$ & $0.11 \times 10^{1}$ & $0.14 \times 10^{1}$ & $0.31 \times 10^{1}$ & $1.10 \times 10^{1}$ \\
\hline 10 & $4.40 \times 10^{1}$ & $4.50 \times 10^{1}$ & $5.30 \times 10^{1}$ & $6.30 \times 10^{1}$ & $0.11 \times 10^{1}$ & $0.57 \times 10^{1}$ \\
\hline 15 & $7.40 \times 10^{1}$ & $7.50 \times 10^{1}$ & $7.60 \times 10^{1}$ & $8.10 \times 10^{1}$ & $0.11 \times 10^{1}$ & $0.20 \times 10^{1}$ \\
\hline 20 & $0.12 \times 10^{1}$ & $0.12 \times 10^{1}$ & $0.12 \times 10^{1}$ & $0.12 \times 10^{1}$ & $0.11 \times 10^{1}$ & $0.16 \times 10^{1}$ \\
\hline 25 & $0.13 \times 10^{1}$ & $0.13 \times 10^{1}$ & $0.14 \times 10^{1}$ & $0.14 \times 10^{1}$ & $0.11 \times 10^{1}$ & $0.14 \times 10^{1}$ \\
\hline 30 & $0.10 \times 10^{1}$ & $0.10 \times 10^{1}$ & $0.13 \times 10^{1}$ & $0.14 \times 10^{1}$ & $0.13 \times 10^{1}$ & $0.12 \times 10^{1}$ \\
\hline 50 & $0.11 \times 10^{1}$ & $0.11 \times 10^{1}$ & $0.11 \times 10^{1}$ & $0.12 \times 10^{1}$ & $0.11 \times 10^{1}$ & $0.11 \times 10^{1}$ \\
\hline 60 & $0.11 \times 10^{1}$ & $0.11 \times 10^{1}$ & $0.11 \times 10^{1}$ & $0.11 \times 10^{1}$ & $0.12 \times 10^{1}$ & $0.15 \times 10^{1}$ \\
\hline 80 & $0.11 \times 10^{1}$ & $0.11 \times 10^{1}$ & $0.11 \times 10^{1}$ & $0.11 \times 10^{1}$ & $0.14 \times 10^{1}$ & $0.16 \times 10^{1}$ \\
\hline 100 & $0.12 \times 10^{1}$ & $0.12 \times 10^{1}$ & $0.12 \times 10^{1}$ & $0.12 \times 10^{1}$ & $0.14 \times 10^{1}$ & $0.15 \times 10^{1}$ \\
\hline 150 & $0.18 \times 10^{1}$ & $0.18 \times 10^{1}$ & $0.18 \times 10^{1}$ & $0.18 \times 10^{1}$ & $0.18 \times 10^{1}$ & $0.18 \times 10^{1}$ \\
\hline 200 & $0.28 \times 10^{1}$ & $0.28 \times 10^{1}$ & $0.28 \times 10^{1}$ & $0.28 \times 10^{1}$ & $0.27 \times 10^{1}$ & $0.70 \times 10^{1}$ \\
\hline 500 & $5.90 \times 10^{1}$ & $5.90 \times 10^{1}$ & $5.90 \times 10^{1}$ & $5.90 \times 10^{1}$ & $5.80 \times 10^{1}$ & $5.80 \times 10^{1}$ \\
\hline 1000 & $2.40 \times 10^{1}$ & $2.40 \times 10^{1}$ & $2.40 \times 10^{1}$ & $2.40 \times 10^{1}$ & $2.40 \times 10^{1}$ & $2.40 \times 10^{1}$ \\
\hline$T_{e} / \rho$ & $10^{-6}$ & $10^{-5}$ & $10^{-4}$ & $10^{-3}$ & $10^{-2}$ & $10^{-1}$ \\
\hline 1 & $1.29 \times 10^{3}$ & $3.11 \times 10^{3}$ & $4.58 \times 10^{3}$ & $6.46 \times 10^{3}$ & $1.24 \times 10^{4}$ & $3.65 \times 10^{4}$ \\
\hline 3 & $8.79 \times 10^{2}$ & $2.58 \times 10^{3}$ & $1.90 \times 10^{3}$ & $6.81 \times 10^{4}$ & $2.37 \times 10^{5}$ & $2.49 \times 10^{5}$ \\
\hline 5 & $2.82 \times 10^{2}$ & $3.70 \times 10^{3}$ & $3.53 \times 10^{4}$ & $1.78 \times 10^{5}$ & $3.79 \times 10^{5}$ & $3.15 \times 10^{5}$ \\
\hline 7 & $9.30 \times 10^{1}$ & $1.03 \times 10^{3}$ & $1.48 \times 10^{4}$ & $9.17 \times 10^{4}$ & $2.78 \times 10^{5}$ & $2.15 \times 10^{5}$ \\
\hline 10 & $2.20 \times 10^{1}$ & $1.77 \times 10^{2}$ & $2.82 \times 10^{3}$ & $2.53 \times 10^{4}$ & $9.69 \times 10^{4}$ & $5.36 \times 10^{4}$ \\
\hline 15 & $0.62 \times 10^{1}$ & $3.10 \times 10^{1}$ & $2.58 \times 10^{2}$ & $2.21 \times 10^{3}$ & $1.15 \times 10^{4}$ & $1.11 \times 10^{4}$ \\
\hline 20 & $0.32 \times 10^{1}$ & $0.73 \times 10^{1}$ & $3.60 \times 10^{1}$ & $3.14 \times 10^{2}$ & $1.78 \times 10^{3}$ & $3.37 \times 10^{3}$ \\
\hline 25 & $0.16 \times 10^{1}$ & $0.24 \times 10^{1}$ & $1.00 \times 10^{1}$ & $8.80 \times 10^{1}$ & $5.65 \times 10^{2}$ & $1.54 \times 10^{3}$ \\
\hline 30 & $9.60 \times 10^{1}$ & $0.12 \times 10^{1}$ & $0.47 \times 10^{1}$ & $3.80 \times 10^{1}$ & $2.60 \times 10^{2}$ & $9.62 \times 10^{2}$ \\
\hline 50 & $9.10 \times 10^{1}$ & $9.30 \times 10^{1}$ & $0.25 \times 10^{1}$ & $1.60 \times 10^{1}$ & $1.47 \times 10^{2}$ & $6.70 \times 10^{2}$ \\
\hline 60 & $0.11 \times 10^{1}$ & $0.10 \times 10^{1}$ & $0.25 \times 10^{1}$ & $1.60 \times 10^{1}$ & $1.35 \times 10^{2}$ & $8.17 \times 10^{2}$ \\
\hline 80 & $0.12 \times 10^{1}$ & $0.12 \times 10^{1}$ & $0.32 \times 10^{1}$ & $1.80 \times 10^{1}$ & $1.39 \times 10^{2}$ & $8.52 \times 10^{2}$ \\
\hline 100 & $0.12 \times 10^{1}$ & $0.14 \times 10^{1}$ & $0.35 \times 10^{1}$ & $1.60 \times 10^{1}$ & $1.15 \times 10^{2}$ & $7.54 \times 10^{2}$ \\
\hline 150 & $0.17 \times 10^{1}$ & $0.20 \times 10^{1}$ & $0.38 \times 10^{1}$ & $1.10 \times 10^{1}$ & $4.50 \times 10$ & $1.79 \times 10^{2}$ \\
\hline 200 & $0.26 \times 10^{1}$ & $0.29 \times 10^{1}$ & $0.39 \times 10^{1}$ & $0.65 \times 10^{1}$ & $1.30 \times 10^{1}$ & $3.60 \times 10^{1}$ \\
\hline 500 & $5.70 \times 10^{1}$ & $5.40 \times 10^{1}$ & $4.70 \times 10^{1}$ & $4.00 \times 10^{1}$ & $3.90 \times 10^{1}$ & $5.60 \times 10^{1}$ \\
\hline 1000 & $2.40 \times 10^{1}$ & $2.30 \times 10^{1}$ & $2.30 \times 10^{1}$ & $2.20 \times 10^{1}$ & $2.20 \times 10^{1}$ & $2.30 \times 10^{1}$ \\
\hline
\end{tabular}


transitions which photon energies that lie in the spectral window of the weighting functions. For this reason we can obtain relative deviation of the mean opacities lower than $10 \%$ in some NLTE plasma conditions, as it is shown, for example, in Table 4 for $80 \mathrm{eV}$ and $10^{-2} \mathrm{~g} \mathrm{~cm}^{-3}$ where the relative deviation of the Rosseland mean opacity is about 5\%. Furthermore, we have represented in Fig. 5 Planck and Rosseland mean absorption coefficients as a function of electron temperature in the range of density of matter given by $10^{-1}-10^{-7} \mathrm{~g} \mathrm{~cm}^{-3}$ (electron densities in the range $10^{16}-10^{22} \mathrm{~cm}^{-3}$ ) calculated using CE, CRSS and SB equations. In general, the SB calculations underestimate the Planck mean absorption coefficients with respect to those obtained from CRSS calculations, increasing their relative deviations either when the density decreases or the temperature increases. On the other hand, at high temperatures, the Rosseland mean absorption coefficients obtained from CRSS and SB calculations tend to the same value. It is due to the scattering Thomson processes, which is dominant at these plasmas conditions, and it is independent on the level populations in our model.

Finally, in Tables 5 and 6 are listed the values of the Planck and Rosseland mean opacities, respectively, in a

\section{Table 7}

Relative differences of the Planck multigroup opacities (in percentage) between the opacities calculated using the CRSS model and the SB equations. The electron temperatures are (a) $30 \mathrm{eV}$ and (b) $50 \mathrm{eV}$. The density of matter is given in $\mathrm{g} \mathrm{cm}^{-3}$ and the range of photon energies $(\Delta E)$ is given in $\mathrm{eV}$.

\begin{tabular}{|c|c|c|c|c|c|c|c|}
\hline \multirow[t]{2}{*}{ Group } & \multirow[t]{2}{*}{$\Delta E$} & \multicolumn{2}{|c|}{$\rho=10^{-1}$} & \multicolumn{2}{|c|}{$\rho=10^{-3}$} & \multicolumn{2}{|c|}{$\rho=10^{-5}$} \\
\hline & & (a) & (b) & (a) & (b) & (a) & (b) \\
\hline 1 & $0-13$ & 0.03 & 1.12 & 0.62 & 9.98 & 19.06 & 59.67 \\
\hline 2 & $13-26$ & 0.06 & 1.11 & 2.16 & 13.82 & 95.98 & $>100$ \\
\hline 3 & $26-39$ & 0.01 & 0.92 & 1.83 & 3.97 & 76.18 & 18.39 \\
\hline 4 & $39-52$ & 0.00 & 1.78 & 7.77 & 19.77 & 99.46 & 77.56 \\
\hline 5 & $52-65$ & 0.00 & 3.77 & 3.24 & 17.85 & 51.95 & 55.42 \\
\hline 6 & $65-78$ & 0.00 & 4.37 & 3.00 & 33.91 & 35.27 & $>100$ \\
\hline 7 & $78-91$ & 0.00 & 4.26 & 3.12 & 23.30 & 18.80 & 79.79 \\
\hline 8 & $91-104$ & 0.00 & 4.24 & 3.00 & 26.40 & 50.72 & $>100$ \\
\hline 9 & $104-117$ & 0.00 & 4.17 & 3.02 & 26.39 & 37.40 & $>100$ \\
\hline 10 & $117-130$ & 0.00 & 4.13 & 3.01 & 27.10 & 37.83 & $>100$ \\
\hline 11 & $130-202$ & 0.01 & 4.05 & 3.03 & 26.60 & 43.85 & $>100$ \\
\hline 12 & $202-275$ & 0.00 & 0.20 & 1.62 & 6.48 & 22.83 & 67.13 \\
\hline 13 & $275-287$ & 0.00 & 1.33 & 0.54 & 14.39 & 33.24 & 52.92 \\
\hline 14 & $287-300$ & 0.00 & 1.71 & 1.01 & 20.23 & 50.89 & 82.12 \\
\hline 15 & $300-312$ & 0.00 & 2.03 & 1.61 & 22.75 & 64.97 & 85.92 \\
\hline 16 & $312-325$ & 0.01 & 1.54 & 1.22 & 19.12 & 59.96 & 79.12 \\
\hline 17 & $325-337$ & 0.00 & 1.27 & 0.95 & 18.48 & 42.38 & 77.09 \\
\hline 18 & $337-350$ & 0.01 & 1.43 & 4.48 & 18.39 & 63.54 & 77.90 \\
\hline 19 & $350-362$ & 0.01 & 0.94 & 1.10 & 19.73 & 64.86 & 84.63 \\
\hline 20 & $362-375$ & 0.04 & 2.75 & 8.45 & 20.80 & $>100$ & 71.84 \\
\hline 21 & $375-387$ & 0.01 & 1.55 & 1.61 & 20.48 & 64.96 & 85.66 \\
\hline 22 & $387-400$ & 0.01 & 1.66 & 1.50 & 21.34 & 64.78 & 84.89 \\
\hline 23 & $400-414$ & 0.01 & 1.51 & 1.56 & 20.79 & 64.98 & 85.22 \\
\hline 24 & $414-428$ & 0.01 & 1.12 & 1.50 & 19.96 & 64.97 & 84.80 \\
\hline 25 & $428-442$ & 0.01 & 0.17 & 0.62 & 5.90 & 23.31 & 47.34 \\
\hline 26 & $442-456$ & 0.01 & 0.46 & 1.56 & 16.77 & 64.97 & 84.03 \\
\hline 27 & $456-470$ & 0.01 & 0.33 & 0.52 & 1.93 & 61.86 & 20.96 \\
\hline 28 & $470-484$ & 0.01 & 0.09 & 0.75 & 1.56 & 7.72 & 12.46 \\
\hline 29 & $484-500$ & 0.01 & 0.08 & 0.27 & 1.52 & 27.72 & 9.21 \\
\hline 30 & $500-600$ & 0.01 & 0.07 & 0.46 & 1.14 & 21.68 & 9.67 \\
\hline
\end{tabular}

wide range of plasma conditions, calculated using the CRSS model implemented in ABAKO.

\subsection{Analysis of the multigroup opacities}

As is well known, the mean opacity or gray atmosphere approximation is a crude way to solve the radiative transport equation and, therefore, it is interesting to analyze the spectrally resolved opacity into the context of the multigroup opacity model. In Table 7 are shown the relative differences of the Planck multigroup opacities, at 30 and $50 \mathrm{eV}$, between the opacities calculated using the CRSS model and the SB equations. The spectrum of photon frequencies has been discretized into 30 energy continuous groups, from 0 to $600 \mathrm{eV}$, assigning a energy group to a given photon and a mean opacity for each energy group from the average of the spectrally resolved opacity. In a similar way in Table 8 we show the relative difference of the Rosseland multigroup opacities. In Figs. 6 and 7 are compared the CRSS and SB calculations of the Rosseland and Planck multigroup opacities vs. the groups of the photon energy at 50 and $10 \mathrm{eV}$ and $10^{-5} \mathrm{~g} \mathrm{~cm}^{-3}$. In general it is detected that the relative deviations obtained in the multigroup opacities are larger than for the mean opacities. However, we can conclude that when the

\section{Table 8}

Relative differences of the Rosseland multigroup opacities (in percentage) between the opacities calculated using the CRSS model and the SB equations. The electron temperatures are (a) $30 \mathrm{eV}$ and (b) $50 \mathrm{eV}$. The density of matter is given in $\mathrm{g} \mathrm{cm}^{-3}$ and the range of photon energies $(\Delta E)$ is given in $\mathrm{eV}$.

\begin{tabular}{|c|c|c|c|c|c|c|c|}
\hline \multirow[t]{2}{*}{ Group } & \multirow[t]{2}{*}{$\Delta E$} & \multicolumn{2}{|c|}{$\rho=10^{-1}$} & \multicolumn{2}{|c|}{$\rho=10^{-3}$} & \multicolumn{2}{|c|}{$\rho=10^{-5}$} \\
\hline & & (a) & (b) & (a) & (b) & (a) & (b) \\
\hline 1 & $0-13$ & 0.10 & 1.11 & 0.54 & 9.97 & 32.82 & 54.55 \\
\hline 2 & $13-26$ & 0.02 & 1.10 & 1.83 & 14.43 & 21.82 & 91.36 \\
\hline 3 & $26-39$ & 0.01 & 0.92 & 0.06 & 5.37 & 12.91 & 59.34 \\
\hline 4 & $39-52$ & 0.00 & 1.97 & 3.12 & 17.05 & 20.20 & 54.75 \\
\hline 5 & $52-65$ & 0.01 & 3.80 & 2.46 & 15.64 & 34.59 & 38.75 \\
\hline 6 & $65-78$ & 0.01 & 4.37 & 2.99 & 28.48 & 68.66 & 86.89 \\
\hline 7 & $78-91$ & 0.01 & 4.25 & 3.12 & 23.48 & 11.94 & 70.94 \\
\hline 8 & $91-104$ & 0.01 & 4.23 & 2.98 & 25.49 & 28.01 & $>100$ \\
\hline 9 & $104-117$ & 0.01 & 4.17 & 3.01 & 26.60 & 29.06 & $>100$ \\
\hline 10 & $117-130$ & 0.01 & 4.12 & 3.02 & 27.06 & 36.14 & $>100$ \\
\hline 11 & $130-202$ & 0.01 & 3.95 & 3.01 & 26.48 & 39.20 & $>100$ \\
\hline 12 & $202-275$ & 0.00 & 0.34 & 1.62 & 7.41 & 19.18 & 59.78 \\
\hline 13 & $275-287$ & 0.00 & 1.30 & 0.49 & 13.88 & 23.81 & 50.74 \\
\hline 14 & $287-300$ & 0.00 & 1.47 & 0.91 & 17.42 & 43.05 & 70.81 \\
\hline 15 & $300-312$ & 0.00 & 1.72 & 1.22 & 19.67 & 57.47 & 81.85 \\
\hline 16 & $312-325$ & 0.01 & 1.45 & 1.08 & 18.24 & 54.49 & 75.68 \\
\hline 17 & $325-337$ & 0.01 & 1.33 & 0.93 & 16.91 & 36.21 & 68.97 \\
\hline 18 & $337-350$ & 0.01 & 1.34 & 0.92 & 16.91 & 16.83 & 70.14 \\
\hline 19 & $350-362$ & 0.01 & 0.71 & 0.09 & 8.18 & 24.37 & 63.00 \\
\hline 20 & $362-375$ & 0.00 & 1.19 & 5.39 & 5.57 & $>100$ & 44.63 \\
\hline 21 & $375-387$ & 0.01 & 1.56 & 1.31 & 20.33 & 59.99 & 83.16 \\
\hline 22 & $387-400$ & 0.01 & 1.66 & 1.53 & 21.34 & 57.62 & 83.70 \\
\hline 23 & $400-414$ & 0.01 & 1.51 & 1.56 & 20.80 & 64.98 & 85.22 \\
\hline 24 & $414-428$ & 0.01 & 1.11 & 1.50 & 20.06 & 64.96 & 84.92 \\
\hline 25 & $428-442$ & 0.01 & 0.13 & 0.73 & 11.67 & 64.11 & 79.27 \\
\hline 26 & $442-456$ & 0.01 & 0.45 & 1.56 & 16.81 & 64.97 & 84.05 \\
\hline 27 & $456-470$ & 0.01 & 0.31 & 0.83 & 10.20 & 64.18 & 77.26 \\
\hline 28 & $470-484$ & 0.01 & 0.09 & 0.78 & 2.57 & 62.14 & 59.22 \\
\hline 29 & $484-500$ & 0.01 & 0.07 & 0.35 & 1.50 & 45.30 & 17.89 \\
\hline 30 & $500-600$ & 0.01 & 0.06 & 0.46 & 1.12 & 21.68 & 9.68 \\
\hline
\end{tabular}



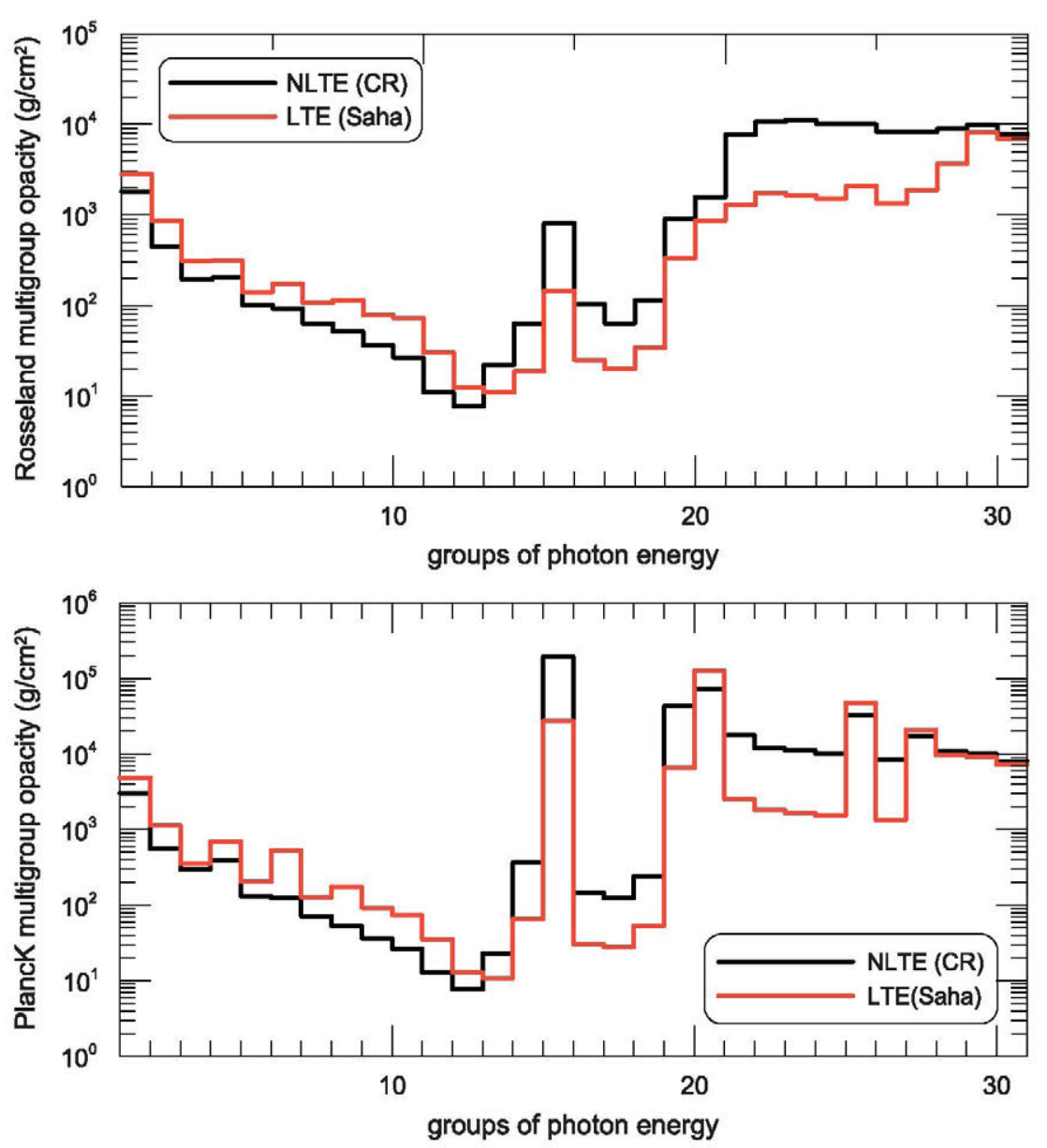

Fig. 6. Comparison between Planck and Rosseland multigroup opacities calculated using the CRSS model and the SB equations. The electron temperature is $50 \mathrm{eV}$ and the density of matter is $10^{-5} \mathrm{~g} \mathrm{~cm}^{-3}$.

criterion in the ion populations is fulfilled, both the relative differences in the Rosseland and Planck mean opacities and multigroup opacities are always less than or equal to the $10 \%$. For example, at $30 \mathrm{eV}$ and $10^{-3} \mathrm{~g} \mathrm{~cm}^{-3}$, where the plasma is in LTE according to Fig. 2, the relative deviations of the Planck and Rosseland mean opacities are $5.2 \%$ and $0.5 \%$, respectively (see (4)) obtaining relative deviations in the multigroup opacities lower than $10 \%$, as it can be observed in (7) and (8). A similar behaviour can be also observed at $50 \mathrm{eV}$ and $10^{-1} \mathrm{~g} \mathrm{~cm}^{-3}$.

On the other hand, according to the map of thermodynamic regimes (Fig. 2) and the values given in Table 4, the carbon plasma is in NLTE at $50 \mathrm{eV}$ and $10^{-2} \mathrm{~g} \mathrm{~cm}^{-3}$ since the relative deviation of the ion populations is 0.21 , and, therefore, the criterion is fulfilled. In this case the relative deviations in the mean opacities are close to $10 \%$ but there is a noticeable number of frequency groups with relative deviation in the multigroup opacities around the $20 \%$. This result is also obtained at $80 \mathrm{eV}$ and $10^{-2} \mathrm{~g} \mathrm{~cm}^{-3}$ where the relative deviation of the ion population is 0.5 , and the plasma can be considered in NLTE, being the relative deviation in the Rosseland mean opacity close to $5 \%$ but for almost all of the frequency groups considered we obtain relative deviations in the multigroup opacities larger than $50 \%$.

\section{Conclusions}

In this work an analysis of the influence of the thermodynamic regime in the calculation of spectrally resolved, mean and multigroup opacities has been presented for optically thin carbon plasmas in a wide range of matter densities and electron temperatures. A criterion based on the relative mean deviations between ion populations calculated using the CRSS and SB equations implemented in the ABAKO code has been proposed. According to the criterion, when the relative differences are lower than $10 \%$, LTE can be assumed. According to this a map of the thermodynamic regimes has been presented. We have checked that, in general, when the criterion is fulfilled, the relative differences in the average ionization are less than or equal to $1 \%$ and in Planck and Rosseland mean opacities less than or equal to $10 \%$. However, for temperatures where the ions in the plasma are only the $\mathrm{H}$-like and the fully stripped ones, in order that the criterion works, the fully stripped ion must not be 

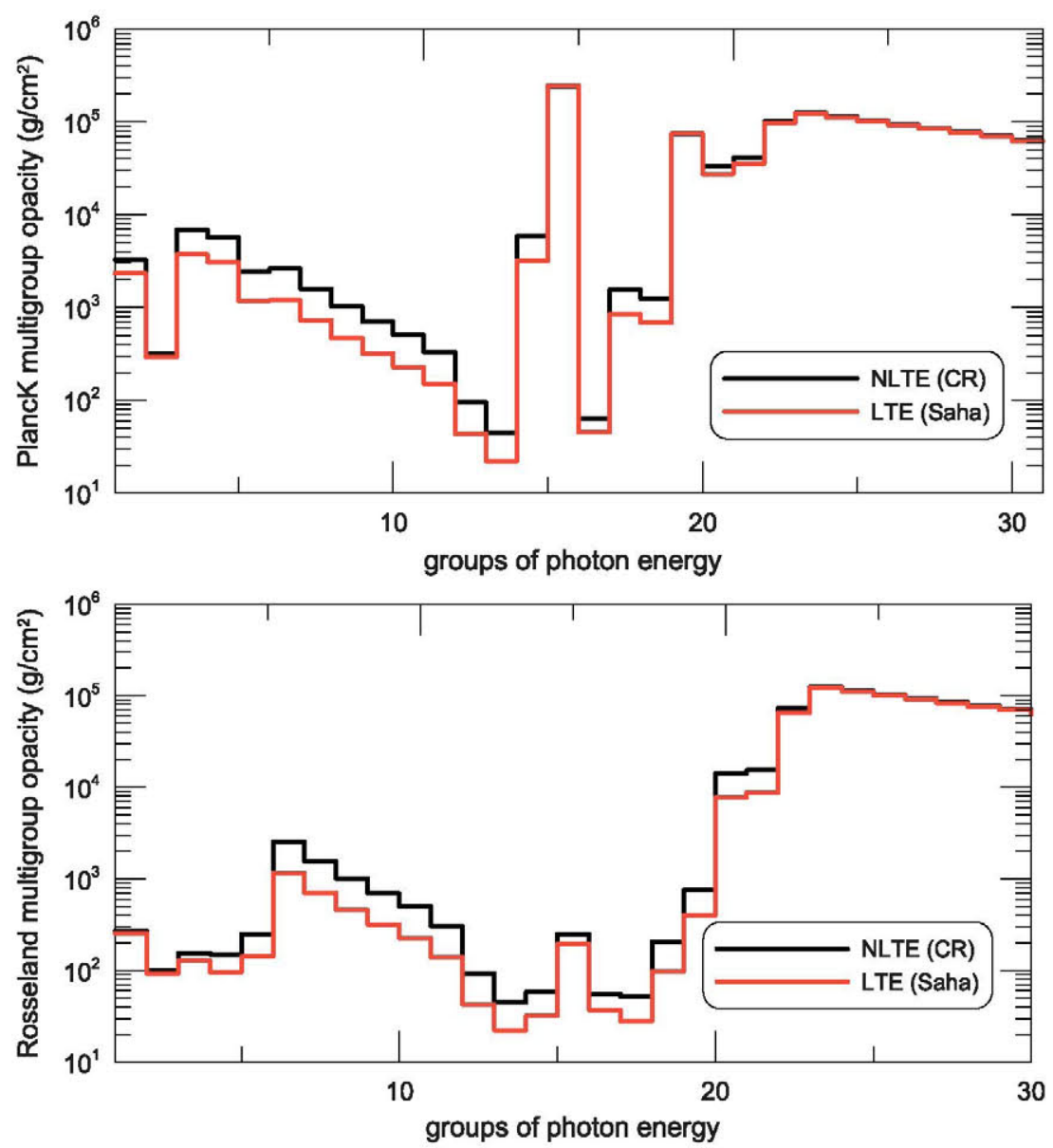

Fig. 7. Comparison between Planck and Rosseland multigroup opacities calculated using the CRSS model and the SB equations. The electron temperature is $10 \mathrm{eV}$ and the density of matter is $10^{-5} \mathrm{~g} \mathrm{~cm}^{-3}$.

included. In particular, for high temperatures, where the fully stripped ion is the only one in the plasma, the criterion is no longer valid. We have proved that when the criterion is satisfied, the spectrally resolved opacities calculated using the CRSS and the SB equations are quite similar. Finally, we have also checked that when the criterion is fulfilled the relative differences in the multigroup opacities are, in general, lower than $10 \%$.

\section{Acknowledgments}

This work has been supported by the Research Project of the Spanish Government ((ENE2009-11208/FTN), by the Research Project of the Government of the Canary Islands (SolSubC200801000057) and also by the Keep in touch Project of the European Union.

\section{References}

[1] Fengtao J, Jiaolong Z, Jianmin Y, Guoxing H, Zeqing W, Jun Y, et al. L to $\mathrm{M}$ shell transitions and model comparisons for radiative opacities of sodium fluoride plasma. J Quant Spectrosc Radiat Transfer 2005;95:241-53.
[2] Bailey JE, Rochau GA, Mancini RC, Iglesias CA, Macfarlane II, Golovkin IE, et al. Experimental investigations of opacity models for stellar interior, inertial fusion, and high energy density plasmas. Phys. Plasmas 2009;16:058101.

[3] Seaton MJ. Atomic data for opacity calculations 1. General description. J Phys B: At Mol Opt Phys 1987;20:6363-78.

[4] Berrinton KA, Burke PG, Butler K, Seaton MJ, Storey PJ, Taylor KT, et al. Atomic data for opacity calculations 2. Computing methods. J Phys B: At Mol Opt Phys 1987;20:6379-97.

[5] Hummer DG, Berrinton KA, Eissner W, Pradhan AK, Saraph HE, Tully JA. Atomic data from the Iron Project. 1. Goals and methods. Astron Astrophys 1993;279:298-309.

[6] Opacity Project Team, The opacity project, vols. 1 and 2. Bristol: Institute of Physics Publishing; 1995.

[7] Zeng J, Yuan J. Detailed-term-accounting approximation calculations of the radiative opacity of aluminum plasma: a systematic study. Phys Rev E 2002;66:0164011.

[8] Serduke FID, Mnguez E, Davidson SI, Iglesias CA. WorkOp-IV summary: lessons from iron opacities. J Quant Spectrosc Radiat Transfer 2000;65:527-41.

[9] Peyrusse 0 . On the superconfiguration approach to model NLTE plasma emission. J Quant Spectrosc Radiat Transfer 2001;71:571-9.

[10] Bar-Shalom A, Klapisc HM, Oreg J. HULLAC, an integrated computer package for atomic processes in plasmas. $J$ Quant Spectrosc Radiat Transfer 2001;71:169-88.

[11] Bowen C. NLTE emissivities via ionisation temperature. J Quant Spectrosc Radiat Transfer 2001;71:201-14.

[12] Iglesias CA, Chen MH, Sonnad V, Wilson BG. A new detailed accounting opacity code for mid-Z elements: TOPAZ. J Quant Spectrosc Radiat Transfer 2003;81:227-36. 
[13] Bowen C, Decoster A, Fontes CJ, Fournier KB, Peyrusse O, Ralchenko YV. Review of the NLTE emissivities code comparison virtual workshop. J Quant Spectrosc Radiat Transfer 2003;81:71-84.

[14] Yuan J, Haynes DA, Peterson RR, Moses GA. Flexible databasedriven opacity and spectrum calculations. J Quant Spectrosc Radiat Transfer 2003;81:513-20.

[15] Rubiano JG, Florido R, Rodrguez R, Gil JM, Martel P, Mnguez E. Calculation of the radiative opacity of laser-produced plasmas using a new relativistic-screened hydrogenic model. J Quant Spectrosc Radiat Transfer 2004;83:159-82.

[16] Chung HK, Chen MH, Morgan WL, Ralchenko Y, Lee RW. FLYCHK: generalized population kinetics and spectral model for rapid spectroscopic analysis for all elements. High Energy Density Phys $2005 ; 1: 3-12$

[17] Hakel P, Sherrill ME, Mazevet S, Abdallah J, Colgan J, Kilcrease DP, et al. The new los alamos opacity code atomic. J Quant Spectrosc Radiat Transfer 2006:99(1-3):265-71.

[18] Wu Z, Pang J, Yan J. Opacity calculations for high-Z plasmas in nonlocal thermodynamic equilibrium. J Quant Spectrosc Radiat Transfer 2006;102:402-8.

[19] Yuan J, Moses GA. YAC: a code using the detailed accounting model for all-Z elements. J Quant Spectrosc Radiat Transfer 2006;99: 697-711.

[20] Mazevet S, Abdallah J. Mixed UTA and detailed line treatment for mid-z opacity and spectral calculations. J Phys B: At Mol Opt Phys 2006;39:3419-29.

[21] Rodriguez R, Gil JM, Florido R, Rubiano JG, Martel P, Minguez E. Code to calculate optical properties for plasmas in a wide range of densities. J Phys IV 2006;133:981-4.

[22] Rodriguez R, Florido R, Gil JM, Rubiano JG, Martel P, Minguez E Rapcal code: a computational package to compute radiative properties for optically thin and thick low and high $=z$ plasmas in a wide range of density and temperature. Laser Part Beams 2008;26: 433-48.

[23] Abdallah Jr. J, Kilcrease DP, Magee NH, Mazevet S, Hakel P, Sherrill M. Spectral line strength binning method for opacity calculations. High Energy Density Phys 2007;3:309-13.

[24] Abadallah Jr. J, Batani D, Desai T, Lucchini G, Faenov A, Pikuz T, et al. High resolution $\mathrm{X}$-ray emission spectra from picosecond laser irradiated Ge targets. Laser Part Beams 2007;25:245-52.

[25] Hansen S, Bauche J, Bauche-Arnoult C, Gu MF. Hybrid atomic models for spectroscopic plasma diagnostics. High Energy Density Phys 2007;3:109-14.

[26] Florido R, Rodrguez R, Gil JM, Rubiano JG, Martel P, Minguez E, et al Modeling of population kinetics of plasmas that are not in local thermodynamic equilibrium, using a versatile collisional-radiative model based on analytical rates. Phys Rev E 2009;80:056402.

[27] Gil JM, Rodriguez R, Florido R, Rubiano JG, Martel P, Minguez E. Determination of corona, LTE and NLTE regimes of optically thin carbon plasmas. Laser Part Beams 2008;26:21-31.

[28] Rodriguez R, Florido R, Gil JM, Rubiano JG, Suarez D, Martel P, et al. Commun Comput Phys 2010;8:185.

[29] GU MF. Can J Phys 2008;86:675.

[30] Bowen C, Lee RW, Ralchenko Yu. Comparing plasma population kinetics codes: review of the NLTE-3 kinetics workshop. I Quant Spectrosc Radiat Transfer 2006;99:102-19.
[31] Rubiano JG, Florido R, Bowen C, Lee RW, Ralchenko Y. Review of the 4 th NLTE code comparison workshop. High Energy Density Phys 2007:3:225-32.

[32] Fontes CJ, Abdallah J, Bowen C, Lee RW, Ralchenko Y. Review of the NLTE-5 kinetics workshop. High Energy Density Phys 2009;5 $15-22$.

[33] Lotz W. Electron-impact ionization cross sections and ionization coefficients for atoms and ions from hydrogen to calcium. Z Phys $1968 ; 216: 241-7$.

[34] Van Regemorter HV. Rate of collisional excitation in stellar atmospheres. Astrophys J 1962;136:906-15.

[35] Kramers HA. On the theory of X-ray absorption and of the continuous X-ray spectrum. Philos Mag 1923;46:836-71.

[36] Griem HR. In: Principles of plasma spectroscopy. Cambridge: Cambridge University Press; 1997. pp. 177-179.

[37] Wu ZQ Zhang BA, Qiu YB. Effect of excitation-autoionization in non-local thermodynamic equilibrium plasmas. Chin Phys 2000;9: $519-22$

[38] Jacobs VL. Autoionization phenomena in plasma radiation processes. J Quant Spectrosc Radiat Transfer 1995;54:195-205.

[39] Rodriguez R, Florido R, Gil JM, Rubiano JG, Martel P, Mnguez E Influence of the atomic description and configuration interaction effects on collisional-radiative calculations of low ionized carbon plasmas. J Quant Spectrosc Radiat Transfer 2009;110:2191-207.

[40] Stewart JC, Pyatt KD. Lowering of ionization potentials in plasmas. Astrophys J 1966;144:1203-11.

[41] Cowan RD. In: The theory of atomic structure. Berkeley: University of California Press; 1981

[42] Dimitrijevic MS, Konjevic N. Simple estimates for Stark-broadening of ion lines in stellar plasmas. Astron Astrophys 1987;172:345-9.

[43] Rutten RJ. Radiative transfer in stellar atmospheres, Lectures Notes, 8th ed., Utretch: Utretch University; 2003.

[44] Rose J. Calculation of the radiative opacity of laser-produced plasma. J Phys B: At Mol Opt Phys 1992;28:1667-81.

[45] Griem HR. Validity of local thermodynamic equilibrium in plasma spectroscopy. Phys Rev 1963;131:1170-6.

[46] Salzmann D. In: Atomic physics in hot plasmas. New York: Oxford University Press; 1998.

[47] Rodriguez R, Gil JM, Florido R, Rubiano JG, Mendoza MA, Martel P, et al. Determination and analysis of the thermodynamic regimes of xenon plasmas. Contrib Plasma Phys 2011:51:863-76.

[48] Rodriguez R, Gil JM, Florido R, Rubiano JG, Mendoza MA, Martel P, et al. Determination of the average ionization and thermodynamic regimes of xenon plasmas with an application to the characterization of blast waves launched in xenon clusters. High Energy Density Phys 2011;7:71-6.

[49] Colgan J, Fontes CJ, Abdallah J. Collisional-radiative studies of carbon plasmas. High Energy Density Phys 2006;2:90-6.

[50] Magee NH, Abdallah J, Clark REH. Atomic structure calculations and new Los Alamos astrophysical opacities. Astron. Astrophys. 1995;78:51-6.

[51] Nikiforov AF, Novikov VG, Uvarov VB. In: Quantum-statistical model of hot dense matter and methods for computation opacity and equation of state. Moscow: Fizmattlit; 2000. 